\title{
The Cauchy problem for the Landau-Lifshitz-Gilbert equation in BMO and self-similar solutions
}

\author{
Susana Gutiérrez ${ }^{1}$ and André de Laire ${ }^{2}$
}

\begin{abstract}
We prove a global well-posedness result for the Landau-Lifshitz equation with Gilbert damping provided that the BMO semi-norm of the initial data is small. As a consequence, we deduce the existence of self-similar solutions in any dimension. In the one-dimensional case, we characterize the self-similar solutions associated with an initial data given by some $\left(\mathbb{S}^{2}\right.$-valued) step function and establish their stability. We also show the existence of multiple solutions if the damping is strong enough.

Our arguments rely on the study of a dissipative quasilinear Schrödinger equation obtained via the stereographic projection and techniques introduced by Koch and Tataru.

Keywords and phrases: Landau-Lifshitz-Gilbert equation, global well-posedness, discontinuous initial data, stability, self-similar solutions, dissipative Schrödinger equation, complex Ginzburg-Landau equation, ferromagnetic spin chain, heat-flow for harmonic maps.
\end{abstract}

2010 Mathematics Subject Classification: 35R05, 35Q60, 35A01, 35C06, 35B35, 35Q55, 35Q56, 35A02, 53C44.

\section{Contents}

$1 \quad$ Introduction and main results $\quad 2$

2 The Cauchy problem $\quad 6$

2.1 The Cauchy problem for a dissipative quasilinear Schrödinger equation . . . . . . 6

2.2 The Cauchy problem for the LLG equation . . . . . . . . . . . . . . 15

3 Applications $\quad 22$

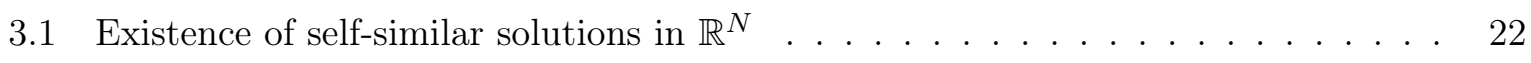

3.2 The Cauchy problem for the one-dimensional LLG equation with a jump initial

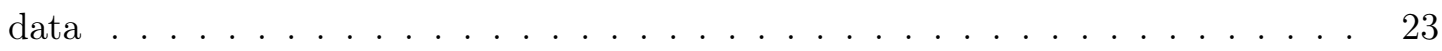

3.2.1 Existence, uniqueness and stability. Proof of Theorem $1.2 \ldots \ldots$. . . . 24

3.2 .2 Multiplicity of solutions. Proof of Theorem $1.3 \ldots \ldots \ldots$

3.3 A singular solution for a nonlocal Schrödinger equation . . . . . . . . . . . . 30

\section{Appendix}

\footnotetext{
${ }^{1}$ School of Mathematics, University of Birmingham, Edgbaston, Birmingham, B15 2TT, United Kingdom. E-mail: s.gutierrez@bham.ac.uk

${ }^{2}$ Univ. Lille, CNRS, Inria, UMR 8524, Laboratoire Paul Painlevé, F-59000 Lille, France.

E-mail: andre.de-laire@univ-lille.fr
} 


\section{Introduction and main results}

We consider the Landau-Lifshitz-Gilbert (LLG) equation

$$
\partial_{t} \boldsymbol{m}=\beta \boldsymbol{m} \times \Delta \boldsymbol{m}-\alpha \boldsymbol{m} \times(\boldsymbol{m} \times \Delta \boldsymbol{m}), \quad \text { on } \mathbb{R}^{N} \times \mathbb{R}^{+},
$$

where $\boldsymbol{m}=\left(m_{1}, m_{2}, m_{3}\right): \mathbb{R}^{N} \times \mathbb{R}^{+} \longrightarrow \mathbb{S}^{2}$ is the spin vector, $\beta \geq 0, \alpha \geq 0, \times$ denotes the usual cross-product in $\mathbb{R}^{3}$, and $\mathbb{S}^{2}$ is the unit sphere in $\mathbb{R}^{3}$. This model introduced by Landau and Lifshitz describes the dynamics for the spin in ferromagnetic materials $[26,16]$ and constitutes a fundamental equation in the magnetic recording industry [36]. The parameters $\beta \geq 0$ and $\alpha \geq 0$ are respectively the so-called exchange constant and Gilbert damping, and take into account the exchange of energy in the system and the effect of damping on the spin chain. Note that, by performing a time-scaling, we assume w.l.o.g. that

$$
\alpha \in[0,1] \text { and } \beta=\sqrt{1-\alpha^{2}} .
$$

The Landau-Lifshitz family of equations includes as special cases the well-known heat-flow for harmonic maps and the Schrödinger map equation onto the 2 -sphere. In the limit case $\beta=0$ (and so $\alpha=1$ ) the LLG equation reduces to the heat-flow equation for harmonic maps

$$
\partial_{t} \boldsymbol{m}-\Delta \boldsymbol{m}=|\nabla \boldsymbol{m}|^{2} \boldsymbol{m}, \quad \text { on } \mathbb{R}^{N} \times \mathbb{R}^{+} .
$$

The case when $\alpha=0$ (i.e. no dissipation/damping) corresponds to the Schrödinger map equation

$$
\partial_{t} \boldsymbol{m}=\boldsymbol{m} \times \Delta \boldsymbol{m}, \quad \text { on } \mathbb{R}^{N} \times \mathbb{R}^{+} .
$$

In the one-dimensional case $N=1$, we established in [17] the existence and asymptotics of the family $\left\{\boldsymbol{m}_{c, \alpha}\right\}_{c>0}$ of self-similar solutions of $\left(\mathrm{LLG}_{\alpha}\right)$ for any fixed $\alpha \in[0,1]$, extending the results in Gutiérrez, Rivas and Vega [18] in the setting of the Schrödinger map equation and related binormal flow equation. The motivation for the results presented in this paper first originated from the desire to study further properties of the self-similar solutions found in [17], and in particular their stability. In the case $\alpha=0$, the stability of the self-similar solutions of the Schrödinger map has been considered in the series of papers by Banica and Vega [5, 6, 7], but no stability result is known for these solutions in the presence of damping, i.e. $\alpha>0$. One of the key ingredients in the analysis given by Banica and Vega is the reversibility in time of the equation in the absence of damping. However, since $\left(L_{L} G_{\alpha}\right)$ is a dissipative equation for $\alpha>0$, this property is no longer available and a new approach is needed.

In the one-dimensional case and for fixed $\alpha \in[0,1]$, the self-similar solutions of $\left(\mathrm{LLG}_{\alpha}\right)$ constitute a uniparametric family $\left\{\boldsymbol{m}_{c, \alpha}\right\}_{c>0}$ where $\boldsymbol{m}_{c, \alpha}$ is defined by

$$
\boldsymbol{m}_{c, \alpha}(x, t)=\boldsymbol{f}\left(\frac{x}{\sqrt{t}}\right)
$$

for some profile $\boldsymbol{f}: \mathbb{R} \longrightarrow \mathbb{S}^{2}$, and is associated with an initial condition given by a step function (at least when $c$ is small) of the form

$$
\boldsymbol{m}_{c, \alpha}^{0}:=\boldsymbol{A}_{c, \alpha}^{+} \chi_{\mathbb{R}^{+}}+\boldsymbol{A}_{c, \alpha}^{-} \chi_{\mathbb{R}^{-}},
$$

where $\boldsymbol{A}_{c, \alpha}^{ \pm}$are certain unitary vectors and $\chi_{E}$ denotes the characteristic function of a set $E$. In particular, when $\alpha>0$, the Dirichlet energy associated with the solutions $\boldsymbol{m}_{c, \alpha}$ given by

$$
\left\|\nabla \boldsymbol{m}_{c, \alpha}(\cdot, t)\right\|_{L^{2}}^{2}=c^{2}\left(\frac{2 \pi}{\alpha t}\right)^{1 / 2}, \quad t>0
$$


diverges as $t \rightarrow 0^{+3}$.

A first natural question in the study of the stability properties of the family of solutions $\left\{\boldsymbol{m}_{c, \alpha}\right\}_{c>0}$ is whether or not it is possible to develop a well-posedness theory for the Cauchy problem for $\left(\mathrm{LLG}_{\alpha}\right)$ in a functional framework that allows us to handle initial conditions of the type (1.1). In view of (1.1) and (1.2), such a framework should allow some "rough" functions (i.e. function spaces beyond the "classical" energy ones) and step functions.

A few remarks about previously known results in this setting are in order. In the case $\alpha>0$, global well-posedness results for $\left(\mathrm{LLG}_{\alpha}\right)$ have been established in $N \geq 2$ by Melcher [31] and by Lin, Lai and Wang [30] for initial conditions with a smallness condition on the gradient in the $L^{N}\left(\mathbb{R}^{N}\right)$ and the Morrey $M^{2,2}\left(\mathbb{R}^{N}\right)$ norm ${ }^{4}$, respectively. Therefore these results do not apply to the initial condition $\boldsymbol{m}_{c, \alpha}^{0}$. When $\alpha=1$, global well-posedness results for the heat flow for harmonic maps (HFHM) have been obtained by Koch and Lamm [22] for an initial condition $L^{\infty}$-close to a point and improved to an initial data with small BMO semi-norm by Wang [35]. The ideas used in [22] and [35] rely on techniques introduced by Koch and Tataru [23] for the Navier-Stokes equation. Since $\boldsymbol{m}_{c, \alpha}^{0}$ has a small BMO semi-norm if $c$ is small, the results in [35] apply to the case $\alpha=1$.

There are two main purposes in this paper. The first one is to adapt and extend the techniques developed in $[22,23,35]$ to prove a global well-posedness result for $\left(\operatorname{LLG}_{\alpha}\right)$ with $\alpha \in(0,1]$ for data $\boldsymbol{m}^{0}$ in $L^{\infty}\left(\mathbb{R}^{N} ; \mathbb{S}^{2}\right)$ with small BMO semi-norm. The second one is to apply this result to establish the stability of the family of self-similar solutions $\left\{\boldsymbol{m}_{c, \alpha}\right\}_{c>0}$ found in [17] and derive further properties for these solutions. In particular, a further understanding of the properties of the functions $\boldsymbol{m}_{c, \alpha}$ will allow us to prove the existence of multiple smooth solutions of (LLG $\mathrm{G}_{\alpha}$ associated with the same initial condition, provided that $\alpha$ is close to one.

In order to state the first of our results, we introduce the function space $X$ as follows:

$$
X=\left\{v: \mathbb{R}^{n} \times \mathbb{R}^{+} \rightarrow \mathbb{R}^{3}: v, \nabla v \in L_{\mathrm{loc}}^{1}\left(\mathbb{R}^{N} \times \mathbb{R}^{+}\right) \text {and }\|v\|_{X}:=\sup _{t>0}\|v(t)\|_{L^{\infty}}+[v]_{X}<\infty\right\}
$$

where

$$
[v]_{X}:=\sup _{t>0} \sqrt{t}\|\nabla v\|_{L^{\infty}}+\sup _{\substack{x \in \mathbb{R}^{N} \\ r>0}}\left(\frac{1}{r^{N}} \int_{B_{r}(x) \times\left[0, r^{2}\right]}|\nabla v(y, t)|^{2} d t d y\right)^{\frac{1}{2}},
$$

and $B_{r}(x)$ denotes the ball with center $x$ and radius $r>0$ in $\mathbb{R}^{N}$. Let us remark that the first term in the definition of $[v]_{X}$ allows to capture a blow-up rate of $1 / \sqrt{t}$ for $\|\nabla v(t)\|_{L^{\infty}}$, as $t \rightarrow 0^{+}$. This is exactly the blow-up rate for the self-similar solutions (see (3.1) and (3.12)). The integral term in $[v]_{X}$ is associated with the space BMO as explained in Subsection 2.1, and it is also well adapted to the self-similar solutions (see Proposition 3.4 and its proof).

We can now state the following (global) well-posedness result for the Cauchy problem for the LLG equation:

Theorem 1.1. Let $\alpha \in(0,1]$. There exist constants $M_{1}, M_{2}, M_{3}>0$, depending only on $\alpha$ and $N$ such that the following holds. For any $\boldsymbol{m}^{0} \in L^{\infty}\left(\mathbb{R}^{N} ; \mathbb{S}^{2}\right), \boldsymbol{Q} \in \mathbb{S}^{2}, \delta \in(0,2]$ and $\varepsilon_{0}>0$ such that $\varepsilon_{0} \leq M_{1} \delta^{6}$,

$$
\inf _{\mathbb{R}^{N}}\left|\boldsymbol{m}^{0}-\boldsymbol{Q}\right|^{2} \geq 2 \delta \quad \text { and } \quad\left[\boldsymbol{m}^{0}\right]_{B M O} \leq \varepsilon_{0},
$$

there exists a unique solution $\boldsymbol{m} \in X\left(\mathbb{R}^{N} \times \mathbb{R}^{+} ; \mathbb{S}^{2}\right)$ of $\left(\mathrm{LLG}_{\alpha}\right)$ with initial condition $\boldsymbol{m}^{0}$ such that

$$
\inf _{\substack{x \in \mathbb{R}^{N} \\ t>0}}|\boldsymbol{m}(x, t)-\boldsymbol{Q}|^{2} \geq \frac{4}{1+M_{2}^{2}\left(M_{3} \delta^{4}+\delta^{-1}\right)^{2}} \quad \text { and } \quad[\boldsymbol{m}]_{X} \leq 4 M_{2}\left(M_{3} \delta^{4}+8 \delta^{-2} \varepsilon_{0}\right) .
$$

\footnotetext{
${ }^{3}$ We refer the reader to Theorem A.5 in the Appendix and to [17] for precise statements of these results.

${ }^{4}$ See footnote in Section 3.3 for the definition of the Morrey space $M^{2,2}\left(\mathbb{R}^{N}\right)$.
} 
In addition, $\boldsymbol{m}$ is a smooth function belonging to $C^{\infty}\left(\mathbb{R}^{N} \times \mathbb{R}^{+} ; \mathbb{S}^{2}\right)$. Furthermore, assume that $\boldsymbol{n}$ is a solution to $\left(\mathrm{LLG}_{\alpha}\right)$ fulfilling (1.4), with initial condition $\boldsymbol{n}^{0}$ satisfying (1.3). Then

$$
\|\boldsymbol{m}-\boldsymbol{n}\|_{X} \leq \frac{120 M_{2}}{\delta^{2}}\left\|\boldsymbol{m}^{0}-\boldsymbol{n}^{0}\right\|_{L^{\infty}} .
$$

As we will see in Section 2, the proof of Theorem 1.1 relies on the use of the stereographic projection to reduce Theorem 1.1 to establish a well-posedness result for the associated dissipative (quasilinear) Schrödinger equation (see Theorem 2.1). In order to be able to apply Theorem 1.1 to the study of both the initial value problem related to the LLG equation with a jump initial condition, and the stability of the self-similar solutions found in [17], we will need a more quantitative version of this result. A more refined version of Theorem 1.1 will be stated in Theorem 2.9 in Subsection 2.2.

Theorem 1.1 (or more precisely Theorem 2.9) has two important consequences for the Cauchy problem related to $\left(\mathrm{LLG}_{\alpha}\right)$ in one dimension:

$$
\left\{\begin{array}{l}
\partial_{t} \boldsymbol{m}=\beta \boldsymbol{m} \times \partial_{x x} \boldsymbol{m}-\alpha \boldsymbol{m} \times\left(\boldsymbol{m} \times \partial_{x x} \boldsymbol{m}\right), \quad \text { on } \quad \mathbb{R} \times \mathbb{R}^{+}, \\
\boldsymbol{m}_{\boldsymbol{A}^{ \pm}}^{0}:=\boldsymbol{A}^{+} \chi_{\mathbb{R}^{+}}+\boldsymbol{A}^{-} \chi_{\mathbb{R}^{-}},
\end{array}\right.
$$

where $\boldsymbol{A}^{ \pm}$are two given unitary vectors such that the angle between $\boldsymbol{A}^{+}$and $\boldsymbol{A}^{-}$is sufficiently small:

(a) From the uniqueness statement in Theorem 1.1, we can deduce that the solution to (1.6) provided by Theorem 1.1 is a rotation of a self-similar solution $\boldsymbol{m}_{c, \alpha}$ for an appropriate value of $c$ (see Theorem 3.3 for a precise statement).

(b) (Stability) From the dependence of the solution with respect to the initial data established in (1.5) and the analysis of the $1 d$-self-similar solutions $\boldsymbol{m}_{c, \alpha}$ carried out in [17], we obtain the following stability result: For any given $\boldsymbol{m}^{0} \in \mathbb{S}^{2}$ satisfying (1.3) and close enough to $\boldsymbol{m}_{\boldsymbol{A}^{ \pm}}^{0}$, the solution $\boldsymbol{m}$ of $\left(\mathrm{LLG}_{\alpha}\right)$ associated with $\boldsymbol{m}^{0}$ given by Theorem 1.1 must remain close to a rotation of a self-similar solution $\boldsymbol{m}_{c, \alpha}$, for some $c>0$. In particular, $\boldsymbol{m}$ remains close to a self-similar solution.

The precise statement is provided in the following theorem.

Theorem 1.2. Let $\alpha \in(0,1]$. There exist constants $L_{1}, L_{2}, L_{3}>0, \delta^{*} \in(-1,0), \vartheta^{*}>0$ such that the following holds. Let $\boldsymbol{A}^{+}, \boldsymbol{A}^{-} \in \mathbb{S}^{2}$ with angle $\vartheta$ between them. If

$$
0<\vartheta \leq \vartheta^{*}
$$

then there is $c>0$ such that for every $\boldsymbol{m}^{0}$ satisfying

$$
\left\|\boldsymbol{m}^{0}-\boldsymbol{m}_{\boldsymbol{A}^{ \pm}}^{0}\right\|_{L^{\infty}} \leq \frac{c \sqrt{\pi}}{2 \sqrt{\alpha}}
$$

there exists $\mathcal{R} \in S O(3)$, depending only on $\boldsymbol{A}^{+}, \boldsymbol{A}^{-}, \alpha$ and $c$, such that there is a unique global smooth solution $\boldsymbol{m}$ of $\left(\mathrm{LLG}_{\alpha}\right)$ with initial condition $\boldsymbol{m}^{0}$ that satisfies

$$
\inf _{\substack{x \in \mathbb{R} \\ t>0}}(\mathcal{R} \boldsymbol{m})_{3}(x, t) \geq \delta^{*} \quad \text { and } \quad[\boldsymbol{m}]_{X} \leq L_{1}+L_{2} c .
$$

Moreover,

$$
\left\|\boldsymbol{m}-\mathcal{R} \boldsymbol{m}_{c, \alpha}\right\|_{X} \leq L_{3}\left\|\boldsymbol{m}^{0}-\boldsymbol{m}_{\boldsymbol{A}^{ \pm}}^{0}\right\|_{L^{\infty}} .
$$


In particular,

$$
\left\|\partial_{x} \boldsymbol{m}-\partial_{x} \mathcal{R} \boldsymbol{m}_{c, \alpha}\right\|_{L^{\infty}} \leq \frac{L_{3}}{\sqrt{t}}\left\|\boldsymbol{m}^{0}-\boldsymbol{m}_{\boldsymbol{A}^{ \pm}}^{0}\right\|_{L^{\infty}}
$$

for all $t>0$.

Notice that Theorem 1.2 provides the existence of a unique solution in the set defined by the conditions (1.7), and hence it does not exclude the possibility of the existence of other solutions not satisfying these conditions. In fact, as we will see in Theorem 1.3 below, one can prove the existence of multiple solutions of the initial value problem (1.6), at least in the case when $\alpha$ is close to 1 .

We point out that our results are valid only for $\alpha>0$. If we let $\alpha \rightarrow 0$, then the constants $M_{1}$ and $M_{3}$ in Theorem 1.1 go to 0 and $M_{2}$ blows up. Indeed, we use that the kernel associated with the Ginzburg-Landau semigroup $e^{(\alpha+i \beta) t \Delta}$ belongs to $L^{1}$ and its exponential decay. Therefore our techniques cannot be generalized (in a simple way) to cover the critical case $\alpha=0$. In particular, we cannot recover the stability results for the self-similar solutions in the case of Schrödinger maps proved by Banica and Vega in $[5,6,7]$.

As mentioned before, in [30] and [31] some global well-posedness results for ( $\left.\mathrm{LLG}_{\alpha}\right)$ with $\alpha \in(0,1]$ were proved for initial conditions with small gradient in $L^{N}\left(\mathbb{R}^{N}\right)$ and $M^{2,2}\left(\mathbb{R}^{N}\right)$, respectively (see footnote in Subsection 3.3 for the definition of the space $M^{2,2}\left(\mathbb{R}^{N}\right)$ ). In view of the embeddings

$$
L^{N}\left(\mathbb{R}^{N}\right) \subset M^{2,2}\left(\mathbb{R}^{N}\right) \subset B M O^{-1}\left(\mathbb{R}^{N}\right),
$$

for $N \geq 2$, Theorem 1.1 can be seen as generalization of these results since it covers the case of less regular initial conditions. The arguments in [30,31] are based on the method of moving frames that produces a covariant complex Ginzburg-Landau equation. In Subsection 3.3 we give more details and discuss the corresponding equation in the one-dimensional case and provide some properties related to the self-similar solutions.

Our existence and uniqueness result given by Theorem 1.1 requires the initial condition to be small in the BMO semi-norm. Without this condition, the solution could develop a singularity in finite time. In fact, in dimensions $N=3,4$, Ding and Wang [13] have proved that for some smooth initial conditions with small (Dirichlet) energy, the associated solutions of $\left(\mathrm{LLG}_{\alpha}\right)$ blow up in finite time.

In the context of the initial value problem (1.6), the smallness condition in the BMO seminorm is equivalent to the smallness of the angle between $\boldsymbol{A}^{+}$and $\boldsymbol{A}^{-}$. As discussed in [17], in the one dimensional case $N=1$ for fixed $\alpha \in(0,1]$ there is some numerical evidence that indicates the existence of multiple (self-similar) solutions associated with the same initial condition of the type in (1.6) (see Figures 2 and 3 in [17]). This suggests that the Cauchy problem for $\left(\mathrm{LLG}_{\alpha}\right)$ with initial condition (1.6) is ill-posed for general $\boldsymbol{A}^{+}$and $\boldsymbol{A}^{-}$unitary vectors.

The following result states that in the case when $\alpha$ is close to 1 , one can actually prove the existence of multiple smooth solutions associated with the same initial condition $\boldsymbol{m}_{\boldsymbol{A}^{ \pm}}^{0}$. Moreover, given any angle $\vartheta \in(0, \pi)$ between two vectors $\boldsymbol{A}^{+}$and $\boldsymbol{A}^{-} \in \mathbb{S}^{2}$, one can generate any number of distinct solutions by considering values of $\alpha$ sufficiently close to 1 .

Theorem 1.3. Let $k \in \mathbb{N}, \boldsymbol{A}^{+}, \boldsymbol{A}^{-} \in \mathbb{S}^{2}$ and let $\vartheta$ be the angle between $\boldsymbol{A}^{+}$and $\boldsymbol{A}^{-}$. If $\vartheta \in(0, \pi)$, then there exists $\alpha_{k} \in(0,1)$ such that for every $\alpha \in\left[\alpha_{k}, 1\right]$ there are at least $k$ distinct smooth self-similar solutions $\left\{\boldsymbol{m}_{j}\right\}_{j=1}^{k}$ in $X\left(\mathbb{R} \times \mathbb{R}^{+} ; \mathbb{S}^{2}\right)$ of $\left(\mathrm{LLG}_{\alpha}\right)$ with initial condition $\boldsymbol{m}_{\boldsymbol{A}^{ \pm}}^{0}$. These solutions are characterized by a strictly increasing sequence of values $\left\{c_{j}\right\}_{j=1}^{k}$, with $c_{k} \rightarrow \infty$ as $k \rightarrow \infty$, such that

$$
\boldsymbol{m}_{j}=\mathcal{R}_{j} \boldsymbol{m}_{c_{j}, \alpha}
$$


where $\mathcal{R}_{j} \in S O(3)$. In particular

$$
\sqrt{t}\left\|\partial_{x} \boldsymbol{m}_{j}(\cdot, t)\right\|_{L^{\infty}}=c_{j}, \quad \text { for all } t>0 .
$$

Furthermore, if $\alpha=1$ and $\vartheta \in[0, \pi]$, then there is an infinite number of distinct smooth selfsimilar solutions $\left\{\boldsymbol{m}_{j}\right\}_{j \geq 1}$ in $X\left(\mathbb{R} \times \mathbb{R}^{+} ; \mathbb{S}^{2}\right)$ of $\left(\mathrm{LLG}_{\alpha}\right)$ with initial condition $\boldsymbol{m}_{\boldsymbol{A}^{ \pm}}^{0}$. These solutions are also characterized by a sequence $\left\{c_{j}\right\}_{j=1}^{\infty}$ such that (1.8) and (1.9) are satisfied. This sequence is explicitly given by

$$
c_{2 \ell+1}=\ell \sqrt{\pi}-\frac{\vartheta}{2 \sqrt{\pi}}, \quad c_{2 \ell}=\ell \sqrt{\pi}+\frac{\vartheta}{2 \sqrt{\pi}}, \quad \text { for } \ell \geq 0 .
$$

It is important to remark that in particular Theorem 1.3 asserts that when $\alpha=1$, given $\boldsymbol{A}^{+}, \boldsymbol{A}^{-} \in \mathbb{S}^{2}$ such that $\boldsymbol{A}^{+}=\boldsymbol{A}^{-}$, there exists an infinite number of distinct solutions $\left\{\boldsymbol{m}_{j}\right\}_{j \geq 1}$ in $X\left(\mathbb{R} \times \mathbb{R}^{+} ; \mathbb{S}^{2}\right)$ of $\left(\mathrm{LLG}_{\alpha}\right)$ with initial condition $\boldsymbol{m}_{\boldsymbol{A}^{ \pm}}^{0}$ such that $\left[\boldsymbol{m}_{\boldsymbol{A}^{ \pm}}^{0}\right]_{B M O}=0$. This particular case shows that a condition on the size of $X$-norm of the solution as that given in (1.4) in Theorem 1.1 is necessary for the uniqueness of solution. We recall that for finite energy solutions of (HFHM) there are several nonuniqueness results based on Coron's technique [11] in dimension $N=3$. Alouges and Soyeur [2] successfully adapted this idea to prove the existence of multiple solutions of the $\left(\mathrm{LLG}_{\alpha}\right)$, with $\alpha>0$, for maps $\boldsymbol{m}: \Omega \longrightarrow \mathbb{S}^{2}$, with $\Omega$ a bounded regular domain of $\mathbb{R}^{3}$. In our case, since $\left\{c_{j}\right\}_{j=1}^{k}$ is strictly increasing, we have at least $k$ genuinely different smooth solutions. Notice also that the identity (1.9) implies that the $X$-norm of the solution is large as $j \rightarrow \infty$.

Structure of the paper. This paper is organized as follows: in Section 2 we use the stereographic projection to reduce matters to the study the initial value problem for the resulting dissipative Schrödinger equation, prove its global well-posedness in well-adapted normed spaces, and use this result to establish Theorem 2.9 (a more quantitative version of Theorem 1.1). In Section 3 we focus on the self-similar solutions and we prove Theorems 1.2 and 1.3. In Section 3.3 we discuss some implications of the existence of explicit self-similar solutions for the Schrödinger equation obtained by means of the Hasimoto transformation. Finally, and for the convenience of the reader, we have included some regularity results for the complex Ginzburg-Landau equation and some properties of the self-similar solutions $\boldsymbol{m}_{c, \alpha}$ in the Appendix.

Notations. We write $\mathbb{R}^{+}=(0, \infty)$. Throughout this paper we will assume that $\alpha \in(0,1]$ and the constants can depend on $\alpha$. In the proofs $A \lesssim B$ stands for $A \leq C B$ for some constant $C>0$ depending only on $\alpha$ and $N$. We denote in bold the vector-valued variables.

Since we are interested in $\mathbb{S}^{2}$-valued functions, with a slightly abuse of notation, we denote by $L^{\infty}\left(\mathbb{R}^{N} ; \mathbb{S}^{2}\right)\left(\right.$ resp. $\left.X\left(\mathbb{R}^{N} ; \mathbb{S}^{2}\right)\right)$ the space of function in $L^{\infty}\left(\mathbb{R}^{N} ; \mathbb{R}^{3}\right)\left(\right.$ resp. $\left.X\left(\mathbb{R}^{N} ; \mathbb{R}^{3}\right)\right)$ such that $|\boldsymbol{m}|=1$ a.e. on $\mathbb{R}^{N}$.

\section{The Cauchy problem}

\subsection{The Cauchy problem for a dissipative quasilinear Schrödinger equation}

Our approach to study the Cauchy problem for $\left(\mathrm{LLG}_{\alpha}\right)$ consists in analyzing the Cauchy problem for the associated dissipative quasilinear Schrödinger equation through the stereographic projection, and then "transferring" the results back to the original equation. To this end, we introduce the stereographic projection from the South Pole $\mathcal{P}: \mathbb{S}^{2} \backslash\{(0,0,-1)\} \rightarrow \mathbb{C}$ defined for by

$$
\mathcal{P}(\boldsymbol{m})=\frac{m_{1}+i m_{2}}{1+m_{3}}
$$


Let $\boldsymbol{m}$ be a smooth solution of $\left(\mathrm{LLG}_{\alpha}\right)$ with $m_{3}>-1$, then its stereographic projection $u=$ $\mathcal{P}(\boldsymbol{m})$ satisfies the quasilinear dissipative Schrödinger equation (see e.g. [25] for details)

$$
i u_{t}+(\beta-i \alpha) \Delta u=2(\beta-i \alpha) \frac{\bar{u}(\nabla u)^{2}}{1+|u|^{2}} .
$$

At least formally, the Duhamel formula gives the integral equation:

$$
u(x, t)=S_{\alpha}(t) u^{0}+\int_{0}^{t} S_{\alpha}(t-s) g(u)(s) d s,
$$

where $u^{0}=u(\cdot, 0)$ corresponds to the initial condition,

$$
g(u)=-2 i(\beta-i \alpha) \frac{\bar{u}(\nabla u)^{2}}{1+|u|^{2}}
$$

and $S_{\alpha}(t)$ is the dissipative Schrödinger semigroup (also called the complex Ginzburg-Landau semigroup) given by $S_{\alpha}(t) \phi=e^{(\alpha+i \beta) t \Delta} \phi$, i.e.

$$
\left(S_{\alpha}(t) \phi\right)(x)=\int_{\mathbb{R}^{N}} G_{\alpha}(x-y, t) \phi(y) d y, \quad \text { with } \quad G_{\alpha}(x, t)=\frac{e^{-\frac{|x|^{2}}{4(\alpha+i \beta) t}}}{(4 \pi(\alpha+i \beta) t)^{N / 2}} .
$$

One difficulty in studying (IDNLS) is to handle the term $g(u)$. Taking into account that

$$
|\beta-i \alpha|=1 \text { and } \frac{a}{1+a^{2}} \leq \frac{1}{2}, \quad \text { for all } a \geq 0,
$$

we see that

$$
|g(u)| \leq|\nabla u|^{2},
$$

so we need to control $|\nabla u|^{2}$. Koch and Taratu dealt with a similar problem when studying the well-posedness for the Navier-Stokes equation in [23]. Their approach was to introduce some new spaces related to BMO and $\mathrm{BMO}^{-1}$. Later, Koch and Lamm [22] and Wang [35] have adapted these spaces to study some geometric flows. Following these ideas, we define the Banach spaces

$$
\begin{aligned}
& X\left(\mathbb{R}^{N} \times \mathbb{R}^{+} ; F\right)=\left\{v: \mathbb{R}^{N} \times \mathbb{R}^{+} \rightarrow F: v, \nabla v \in L_{\mathrm{loc}}^{1}\left(\mathbb{R}^{N} \times \mathbb{R}^{+}\right),\|v\|_{X}<\infty\right\} \quad \text { and } \\
& Y\left(\mathbb{R}^{N} \times \mathbb{R}^{+} ; F\right)=\left\{v: \mathbb{R}^{N} \times \mathbb{R}^{+} \rightarrow F: v \in L_{\mathrm{loc}}^{1}\left(\mathbb{R}^{N} \times \mathbb{R}^{+}\right),\|v\|_{Y}<\infty\right\}
\end{aligned}
$$

where

$$
\begin{aligned}
\|v\|_{X} & :=\sup _{t>0}\|v\|_{L^{\infty}}+[v]_{X}, \quad \text { with } \\
{[v]_{X} } & :=\sup _{t>0} \sqrt{t}\|\nabla v\|_{L^{\infty}}+\sup _{\substack{x \in \mathbb{R}^{N} \\
r>0}}\left(\frac{1}{r^{N}} \int_{Q_{r}(x)}|\nabla v(y, t)|^{2} d t d y\right)^{\frac{1}{2}}, \quad \text { and } \\
\|v\|_{Y} & =\sup _{t>0} t\|v\|_{L^{\infty}}+\sup _{\substack{x \in \mathbb{R}^{N} \\
r>0}} \frac{1}{r^{N}} \int_{Q_{r}(x)}|v(y, t)| d t d y .
\end{aligned}
$$

Here $Q_{r}(x)$ denotes the parabolic ball $Q_{r}(x)=B_{r}(x) \times\left[0, r^{2}\right]$ and $F$ is either $\mathbb{C}$ or $\mathbb{R}^{3}$. The absolute value stands for the complex absolute value if $F=\mathbb{C}$ and for the euclidean norm if $F=\mathbb{R}^{3}$. We denote with the same symbol the absolute value in $F$ and $F^{3}$. Here and in the sequel we will omit the domain in the norms and semi-norms when they are taken in the whole space, for example $\|\cdot\|_{L^{p}}$ stands for $\|\cdot\|_{L^{p}\left(\mathbb{R}^{N}\right)}$, for $p \in[1, \infty]$. 
The spaces $X$ and $Y$ are related to the spaces $\mathrm{BMO}\left(\mathbb{R}^{N}\right)$ and $\mathrm{BMO}^{-1}\left(\mathbb{R}^{N}\right)$ and are welladapted to study problems involving the heat semigroup $S_{1}(t)=e^{t \Delta}$. In order to establish the properties of the semigroup $S_{\alpha}(t)$ with $\alpha \in(0,1]$, we introduce the spaces $\mathrm{BMO}_{\alpha}\left(\mathbb{R}^{N}\right)$ and $\mathrm{BMO}_{\alpha}^{-1}\left(\mathbb{R}^{N}\right)$ as the space of distributions $f \in S^{\prime}\left(\mathbb{R}^{N} ; F\right)$ such that the semi-norm and norm given respectively by

$$
\begin{aligned}
{[f]_{B M O_{\alpha}} } & :=\sup _{\substack{x \in \mathbb{R}^{N} \\
r>0}}\left(\frac{1}{r^{N}} \int_{Q_{r}(x)}\left|\nabla S_{\alpha}(t) f\right|^{2} d t d y\right)^{\frac{1}{2}}, \quad \text { and } \\
\|f\|_{B M O_{\alpha}^{-1}} & :=\sup _{\substack{x \in \mathbb{R}^{N} \\
r>0}}\left(\frac{1}{r^{N}} \int_{Q_{r}(x)}\left|S_{\alpha}(t) f\right|^{2} d t d y\right)^{\frac{1}{2}},
\end{aligned}
$$

are finite.

On the one hand, the Carleson measure characterization of BMO functions (see [34, Chapter 4] and $\left[27\right.$, Chapter 10]) yields that for fixed $\alpha \in(0,1], B M O_{\alpha}\left(\mathbb{R}^{N}\right)$ coincides with the classical $\operatorname{BMO}\left(\mathbb{R}^{N}\right)$ space $^{5}$, that is for all $\alpha \in(0,1]$ there exists a constant $\Lambda>0$ depending only on $\alpha$ and $N$ such that

$$
\Lambda[f]_{B M O} \leq[f]_{B M O_{\alpha}} \leq \Lambda^{-1}[f]_{B M O} .
$$

On the other hand, Koch and Tataru proved in [23] that $\mathrm{BMO}^{-1}$ (or equivalently $\mathrm{BMO}_{1}^{-1}$, using our notation) can be characterized as the space of derivatives of functions in BMO. A straightforward generalization of their argument shows that the same result holds for $\mathrm{BMO}_{\alpha}^{-1}$ (see Theorem A.1). Hence, using the Carleson measure characterization theorem, we conclude that $\mathrm{BMO}_{\alpha}^{-1}$ coincides with the space $\mathrm{BMO}^{-1}$ and that there exists a constant $\tilde{\Lambda}>0$, depending only on $\alpha$ and $N$, such that

$$
\tilde{\Lambda}\|f\|_{B M O^{-1}} \leq\|f\|_{B M O_{\alpha}^{-1}} \leq \tilde{\Lambda}^{-1}\|f\|_{B M O^{-1}} .
$$

The above remarks allows us to use several of the estimates proved in $[22,23,35]$ in the case $\alpha=1$ to study the integral equation (IDNLS) by using a fixed-point approach.

Our first result concerns the global well-posedness of the Cauchy problem for (IDNLS) with small initial data in $\operatorname{BMO}\left(\mathbb{R}^{N}\right)$.

Theorem 2.1. Let $\alpha \in(0,1]$. There exist constants $C, K \geq 1$ such that for every $L \geq 0, \varepsilon>0$, and $\rho>0$ satisfying

$$
8 C(\rho+\varepsilon)^{2} \leq \rho
$$

if $u^{0} \in L^{\infty}\left(\mathbb{R}^{N} ; \mathbb{C}\right)$, with

$$
\left\|u^{0}\right\|_{L^{\infty}} \leq L \quad \text { and } \quad\left[u^{0}\right]_{B M O} \leq \varepsilon,
$$

then there exists a unique solution $u \in X\left(\mathbb{R}^{N} \times \mathbb{R}^{+} ; \mathbb{C}\right)$ to (IDNLS) such that

$$
[u]_{X} \leq K(\rho+\varepsilon) .
$$

Moreover,

$$
\text { BMO }\left(\mathbb{R}^{N}\right)=\left\{f: \mathbb{R}^{N} \times[0, \infty) \rightarrow F: f \in L_{\mathrm{loc}}^{1}\left(\mathbb{R}^{N}\right),[f]_{B M O}<\infty\right\},
$$

with the semi-norm

$$
[f]_{B M O}=\sup _{\substack{x \in \mathbb{R}^{N} \\ r>0}} f_{B_{r}(x)}\left|f(y)-f_{x, r}\right| d y,
$$

where $f_{x, r}$ is the average

$$
f_{x, r}=f_{B_{r}(x)} f(y) d y=\frac{1}{\left|B_{r}(x)\right|} \int_{B_{r}(x)} f(y) d y .
$$


(i) $\sup _{t>0}\|u\|_{L^{\infty}} \leq K(\rho+L)$.

(ii) $u \in C^{\infty}\left(\mathbb{R}^{N} \times \mathbb{R}^{+}\right)$and (DNLS) holds pointwise.

(iii) $\lim _{t \rightarrow 0^{+}} u(\cdot, t)=u^{0}$ as tempered distributions. Moreover, for every $\varphi \in S\left(\mathbb{R}^{N}\right)$, we have

$$
\left\|\left(u(\cdot, t)-u^{0}\right) \varphi\right\|_{L^{1}} \rightarrow 0, \quad \text { as } t \rightarrow 0^{+} .
$$

(iv) (Dependence on the initial data) Assume that $u$ and $v$ are respectively solutions to (IDNLS) fulfilling (2.8) with initial conditions $u^{0}$ and $v^{0}$ satisfying (2.7). Then

$$
\|u-v\|_{X} \leq 6 K\left\|u^{0}-v^{0}\right\|_{L^{\infty}} .
$$

Although condition (2.6) appears naturally from the fixed-point used in the proof, it may be no so clear at first glance. To better understand it, let us define for $C>0$

$$
\mathcal{S}(C)=\left\{(\rho, \varepsilon) \in \mathbb{R}^{+} \times \mathbb{R}^{+}: C(\rho+\varepsilon)^{2} \leq \rho\right\} .
$$

We see that if $(\rho, \varepsilon) \in \mathcal{S}(C)$, then $\rho, \varepsilon>0$ and

$$
\varepsilon \leq \frac{\sqrt{\rho}}{\sqrt{C}}-\rho
$$

Therefore the set $\mathcal{S}(C)$ is non-empty and bounded. The shape of this set is depicted in Figure 1 . In particular, we infer from $(2.12)$ that if $(\rho, \varepsilon) \in \mathcal{S}(C)$, then

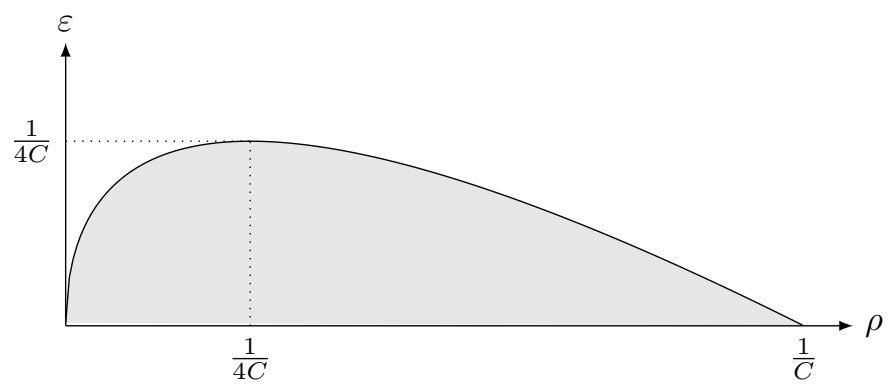

Figure 1: The shape of the set $\mathcal{S}(C)$.

$$
\rho \leq \frac{1}{C} \text { and } \varepsilon \leq \frac{1}{4 C}
$$

In addition, if $\tilde{C} \geq C$, then

$$
\mathcal{S}(\tilde{C}) \subseteq \mathcal{S}(C) .
$$

Moreover, taking for instance $\rho=1 /(32 C)$, Theorem 2.1 asserts that for fixed $\alpha \in(0,1]$, we can take for instance $\varepsilon=1 /(32 C)$ (that depends on $\alpha$ and $N$, but not on the $L^{\infty}$-norm of the initial data) such that for any given initial condition $u^{0} \in L^{\infty}\left(\mathbb{R}^{N}\right)$ with $\left[u^{0}\right]_{B M O} \leq \varepsilon$, there exists a global (smooth) solution $u \in X\left(\mathbb{R}^{N} \times \mathbb{R}^{+} ; \mathbb{C}\right)$ of (DNLS). Notice that $u^{0}$ is allowed to have a large $L^{\infty}$-norm as long as $\left[u^{0}\right]_{B M O}$ is sufficiently small; this is a weaker requirement that asking for the $L^{\infty}$-norm of $u^{0}$ to be sufficiently small, since

$$
[f]_{B M O} \leq 2\|f\|_{L^{\infty}}, \quad \text { for all } f \in L^{\infty}\left(\mathbb{R}^{N}\right) .
$$


Remark 2.2. The smallness condition in (2.8) is necessary for the uniqueness of the solution. As we will see in Subsection 3.2.2, at least in dimension one, it is possible to construct multiple solutions of (IDNLS) in $X\left(\mathbb{R}^{N} \times \mathbb{R}^{+} ; \mathbb{C}\right)$, if $\alpha$ is close enough to 1 .

The aim of this section is to prove Theorem 2.1 using a fixed-point technique. To this pursuit we write (IDNLS) as

$$
u(t)=\mathcal{T}_{u^{0}}(u)(t)
$$

where

$$
\mathcal{T}_{u^{0}}(u)(t)=S_{\alpha}(t) u^{0}+T(g(u))(t) \quad \text { and } \quad T(f)(t)=\int_{0}^{t} S_{\alpha}(t-s) f(s) d s .
$$

In the next lemmas we study the semigroup $S_{\alpha}$ and the operator $T$ to establish that the application $\mathcal{T}_{u^{0}}$ is a contraction on the ball

$$
\mathcal{B}_{\rho}\left(u^{0}\right)=\left\{u \in X\left(\mathbb{R}^{N} \times \mathbb{R}^{+} ; \mathbb{C}\right):\left\|u-S_{\alpha}(t) u^{0}\right\|_{X} \leq \rho\right\},
$$

for some $\rho>0$ depending on the size of the initial data.

Lemma 2.3. There exists $C_{0}>0$ such that for all $f \in B M O_{\alpha}^{-1}\left(\mathbb{R}^{N}\right)$,

$$
\sup _{t>0} \sqrt{t}\left\|S_{\alpha}(t) f\right\|_{L^{\infty}\left(\mathbb{R}^{N}\right)} \leq C_{0}\|f\|_{B M O_{\alpha}^{-1}}
$$

Proof. The proof in the case $\alpha=1$ is done in [27, Lemma 16.1]. For $\alpha \in(0,1)$, decomposing $S_{\alpha}(t)=S_{\alpha}(t-s) S_{\alpha}(s)$ and using the decay properties of the kernel $G_{\alpha}$ associated with the operators $S_{\alpha}(t)$ (see (2.1)), we can check that the same proof still applies.

Lemma 2.4. There exists $C_{1} \geq 1$ such that for all $f \in Y\left(\mathbb{R}^{N} \times \mathbb{R}^{+} ; \mathbb{C}\right)$,

$$
\|T(f)\|_{X} \leq C_{1}\|f\|_{Y}
$$

Proof. Estimate (2.19) can be proved using the arguments given in [23] or [35]. For the convenience of the reader, we sketch the proof following the lines in [35, Lemma 3.1]. By scaling and translation, it suffices to show that

$$
|T(f)(0,1)|+|\nabla T(f)(0,1)|+\left(\int_{Q_{1}(0)}|\nabla T(f)|^{2}\right)^{1 / 2} \lesssim\|f\|_{Y} .
$$

Let $B_{r}=B_{r}(0)$. Setting $W=T(f)$, we have

$$
\begin{aligned}
W(0,1) & =\int_{0}^{1} \int_{\mathbb{R}^{N}} G_{\alpha}(-y, 1-s) f(y, s) d y d s \\
& =\left(\int_{1 / 2}^{1} \int_{\mathbb{R}^{N}}+\int_{0}^{1 / 2} \int_{B_{2}}+\int_{0}^{1 / 2} \int_{\mathbb{R}^{N} \backslash B_{2}}\right) G_{\alpha}(-y, 1-s) f(y, s) d y d s \\
& :=I_{1}+I_{2}+I_{3} .
\end{aligned}
$$

Since $\left|G_{\alpha}(y, 1-s)\right|=\frac{e^{-\frac{\alpha|y|^{2}}{4(1-s)}}}{(4 \pi(1-s))^{N / 2}}$, we obtain

$$
\begin{aligned}
\left|I_{1}\right| & \leq \int_{1 / 2}^{1} \int_{\mathbb{R}^{N}}\left|G_{\alpha}(-y, 1-s) \| f(y, s)\right| d y d s \\
& \leq \sup _{\frac{1}{2} \leq s \leq 1}\|f(s)\|_{L^{\infty}}\left(\int_{\frac{1}{2}}^{1} \int_{\mathbb{R}^{n}}\left|G_{\alpha}(-y, 1-s)\right| d y d s\right) \lesssim\|f\|_{Y},
\end{aligned}
$$




$$
\begin{aligned}
\left|I_{2}\right| & \leq \int_{0}^{1 / 2} \int_{B_{2}}\left|G_{\alpha}(-y, 1-s) \| f(y, s)\right| d y d s \\
& \lesssim \sup _{0 \leq s \leq \frac{1}{2}}\left\|G_{\alpha}(\cdot, 1-s)\right\|_{L^{\infty}\left(\mathbb{R}^{N}\right)} \int_{B_{2} \times\left[0, \frac{1}{2}\right]}|f(y, s)| d y d s \lesssim\|f\|_{Y}
\end{aligned}
$$

and

$$
\begin{aligned}
\left|I_{3}\right| & \leq \int_{0}^{1 / 2} \int_{\mathbb{R}^{N} \backslash B_{2}}\left|G_{\alpha}(-y, 1-s)\right||f(y, s)| d y d s \\
& \leq C \int_{0}^{\frac{1}{2}} \int_{\mathbb{R}^{N} \backslash B_{2}} e^{-\frac{\alpha|y|^{2}}{4}}|f(y, s)| d y d s \\
& \leq C\left(\sum_{k=2}^{\infty} k^{n-1} e^{-\alpha \frac{k^{2}}{4}}\right)\left(\sup _{y \in \mathbb{R}^{N}} \int_{Q_{1}(y)}|f(y, s)| d y d s\right) \lesssim\|f\|_{Y} .
\end{aligned}
$$

The quantity $|\nabla T(f)(0,1)|$ can be bounded in a similar way. The last term in the l.h.s. of $(2.20)$ can be controlled using an energy estimate. Indeed, $W$ satisfies the equation

$$
i \partial_{t} W+(\beta-i \alpha) \Delta W=i f
$$

with initial condition $W(\cdot, 0)=0$. Let $\eta \in C_{0}^{\infty}\left(B_{2}\right)$ be a real-valued cut-off function such that $0 \leq \eta \leq 1$ on $\mathbb{R}^{N}$ and $\eta=1$ on $B_{1}$. By multiplying $(2.21)$ by $-i \eta^{2} \bar{W}$, integrating and taking real part, we get

$$
\frac{1}{2} \partial_{t} \int_{\mathbb{R}^{N}} \eta^{2}|W|^{2}+\alpha \int_{\mathbb{R}^{N}} \eta^{2}|\nabla W|^{2}+2 \operatorname{Re}\left((\alpha+i \beta) \int_{\mathbb{R}^{N}} \eta \nabla \eta \bar{W} \nabla W\right)=\int_{\mathbb{R}^{N}} \eta^{2} \operatorname{Re}(f \bar{W}) .
$$

Using that $|\alpha+i \beta|=1$ and integrating in time between 0 and 1 , it follows that

$$
\frac{1}{2} \int_{\mathbb{R}^{N}} \eta^{2}|W(x, 1)|^{2}+\alpha \int_{\mathbb{R}^{N} \times[0,1]} \eta^{2}|\nabla W|^{2} \leq \int_{\mathbb{R}^{N} \times[0,1]}\left(2 \eta|\nabla \eta||W||\nabla W|+\eta^{2}|f||W|\right) .
$$

From the inequality $a b \leq \varepsilon a^{2}+b^{2} /(4 \varepsilon)$, with $a=\eta|\nabla W|, b=2|\nabla \eta||W|$ and $\varepsilon=\alpha / 2$, we deduce that

$$
\frac{\alpha}{2} \int_{\mathbb{R}^{N} \times[0,1]} \eta^{2}|\nabla W|^{2} \leq \int_{\mathbb{R}^{N} \times[0,1]}\left(\frac{2}{\alpha}|\nabla \eta|^{2}|W|^{2}+\eta^{2}|f||W|\right) .
$$

By the definition of $\eta$, this implies that

$$
\|\nabla W\|_{L^{2}\left(B_{1} \times[0,1]\right)}^{2} \lesssim\|W\|_{L^{\infty}\left(B_{2} \times[0,1]\right)}^{2}+\|W\|_{L^{\infty}\left(B_{2} \times[0,1]\right)}\|f\|_{L^{1}\left(B_{2} \times[0,1]\right)} .
$$

From the first part of the proof, we have

$$
\|W\|_{L^{\infty}\left(B_{2} \times[0,1]\right)} \leq C\|f\|_{Y}
$$

Using also that

$$
\|f\|_{L^{1}\left(B_{2} \times[0,1]\right)} \lesssim\|f\|_{Y},
$$

we conclude from (2.22) that

$$
\|\nabla W\|_{L^{2}\left(B_{1} \times[0,1]\right)} \lesssim\|f\|_{Y},
$$

which finishes the proof. 
Lemma 2.5. Let $\alpha \in(0,1]$ and $\rho, \varepsilon, L>0$. There exists $C_{2} \geq 1$, depending on $\alpha$ and $N$, such that for all $u^{0} \in L^{\infty}\left(\mathbb{R}^{N}\right)$

$$
\left\|S_{\alpha}(t) u^{0}\right\|_{X} \leq C_{2}\left(\left\|u^{0}\right\|_{L^{\infty}}+\left[u^{0}\right]_{B M O}\right) .
$$

If in addition $\left\|u^{0}\right\|_{L^{\infty}} \leq L$ and $\left[u^{0}\right]_{B M O} \leq \varepsilon$, then for all $u \in \mathcal{B}_{\rho}\left(u^{0}\right)$ we have

$$
\sup _{t>0}\|u\|_{L^{\infty}} \leq C_{2}(\rho+L) \quad \text { and } \quad[u]_{X} \leq C_{2}(\rho+\varepsilon) .
$$

Proof. We first control $\left\|S_{\alpha}(t) u^{0}\right\|_{X}$. On the one hand, using the definition of $G_{\alpha}$ and the relation $\alpha^{2}+\beta^{2}=1$, we obtain

$$
\left\|S_{\alpha}(t) u^{0}\right\|_{L^{\infty}}=\left\|G_{\alpha} * u^{0}\right\|_{L^{\infty}} \leq\left\|G_{\alpha}\right\|_{L^{1}}\left\|u^{0}\right\|_{L^{\infty}}=\alpha^{-\frac{N}{2}}\left\|u^{0}\right\|_{L^{\infty}}, \quad \forall t>0 .
$$

Thus

$$
\sup _{t>0}\left\|S_{\alpha}(t) u^{0}\right\|_{L^{\infty}} \leq \alpha^{-\frac{N}{2}}\left\|u^{0}\right\|_{L^{\infty}} .
$$

On the other hand, using Lemma 2.3, Theorem A.1 and (2.4),

$$
\begin{aligned}
{\left[S_{\alpha}(t) u^{0}\right]_{X} } & =\sup _{t>0} \sqrt{t}\left\|\nabla S_{\alpha}(t) u^{0}\right\|_{L^{\infty}}+\sup _{\substack{x \in \mathbb{R}^{N} \\
r>0}}\left(\frac{1}{r^{N}} \int_{Q_{r}(x)}\left|\nabla S_{\alpha}(t) u^{0}\right|^{2} d t d y\right)^{\frac{1}{2}} \\
& \lesssim\left\|\nabla u^{0}\right\|_{B M O_{\alpha}^{-1}}+\left[u^{0}\right]_{B M O_{\alpha}} \\
& \lesssim\left[u^{0}\right]_{B M O_{\alpha}} \\
& \lesssim\left[u^{0}\right]_{B M O} .
\end{aligned}
$$

The estimate in (2.23) follows from (2.25) and (2.26), and we w.l.o.g. can choose $C_{2} \geq 1$. have

Finally, using (2.25), given $u^{0}$ such that $\left\|u^{0}\right\|_{L^{\infty}} \leq L$ and $\left[u^{0}\right]_{B M O} \leq \varepsilon$, for all $u \in \mathcal{B}_{\rho}\left(u^{0}\right)$ we

$$
\|u\|_{L^{\infty}} \leq\left\|u-S_{\alpha}(t) u^{0}\right\|_{L^{\infty}}+\left\|S_{\alpha}(t) u^{0}\right\|_{L^{\infty}} \leq\left\|u-S_{\alpha}(t) u^{0}\right\|_{X}+\left\|S_{\alpha}(t) u^{0}\right\|_{L^{\infty}} \leq C_{2}(\rho+L),
$$

and, using (2.26),

$$
[u]_{X} \leq\left[u-S_{\alpha}(t) u^{0}\right]_{X}+\left[S_{\alpha}(t) u^{0}\right]_{X} \leq\left\|u-S_{\alpha}(t) u^{0}\right\|_{X}+\left[S_{\alpha}(t) u^{0}\right]_{X} \leq C_{2}(\rho+\varepsilon),
$$

which finishes the proof of $(2.24)$.

Now we proceed to bound the nonlinear term

$$
g(u)=-2 i(\beta-i \alpha) \frac{\bar{u}(\nabla u)^{2}}{1+|u|^{2}} .
$$

Lemma 2.6. For all $u \in X\left(\mathbb{R}^{N} \times \mathbb{R}^{+} ; \mathbb{C}\right)$, we have

$$
\|g(u)\|_{Y} \leq[u]_{X}^{2}
$$

Proof. Let $u \in X\left(\mathbb{R}^{N} \times \mathbb{R}^{+} ; \mathbb{C}\right)$. Using $(2.3)$ and the definitions of the norms in $Y$ and $X$, it follows that

$$
\|g(u)\|_{Y} \leq\left(\sup _{t>0} \sqrt{t}\|\nabla u\|_{L^{\infty}}\right)^{2}+\sup _{\substack{x \in \mathbb{R}^{N} \\ r>0}} \frac{1}{r^{N}} \int_{Q_{r}(x)}|\nabla u|^{2} d t d y \leq[u]_{X}^{2} .
$$


Now we have all the estimates to prove that $\mathcal{T}_{u^{0}}$ is a contraction on $\mathcal{B}_{\rho}\left(u^{0}\right)$.

Proposition 2.7. Let $\alpha \in(0,1]$ and $\rho, \varepsilon>0$. Given any $u^{0} \in L^{\infty}\left(\mathbb{R}^{N}\right)$ with $\left[u^{0}\right]_{B M O} \leq \varepsilon$, the operator $\mathcal{T}_{u^{0}}$ given in $(2.17)$ defines a contraction on $\mathcal{B}_{\rho}\left(u^{0}\right)$, whenever $\rho$ and $\varepsilon$ satisfy

$$
8 C_{1} C_{2}^{2}(\rho+\varepsilon)^{2} \leq \rho .
$$

Moreover, for all $u, v \in X\left(\mathbb{R}^{N} \times \mathbb{R}^{+} ; \mathbb{C}\right)$,

$$
\|T(g(u))-T(g(v))\|_{X} \leq C_{1}\left(2[u]_{X}^{2}+[u]_{X}+[v]_{X}\right)\|u-v\|_{X} .
$$

Here, $C_{1} \geq 1$ and $C_{2} \geq 1$ are the constants in Lemmas 2.4 and 2.5, respectively.

Remark 2.8. Using the notation introduced in (2.11), the hypothesis $(2.27)$ means that $(\rho, \varepsilon) \in$ $\mathcal{S}\left(8 C_{1} C_{2}^{2}\right)$. Therefore, by (2.13),

$$
\rho \leq \frac{1}{8 C_{1} C_{2}^{2}}, \text { and } \varepsilon \leq \frac{1}{32 C_{1} C_{2}^{2}},
$$

so $\rho$ and $\varepsilon$ are actually small. Since $C_{1}, C_{2} \geq 1$, we have

$$
C_{2}(\rho+\varepsilon) \leq \frac{5}{32}
$$

Proof. Let $u^{0} \in L^{\infty}\left(\mathbb{R}^{N}\right)$ with $\left\|u^{0}\right\|_{L^{\infty}} \leq L$ and $\left[u^{0}\right]_{B M O} \leq \varepsilon$, and $u \in \mathcal{B}_{\rho}\left(u^{0}\right)$. Using Lemma 2.4, Lemma 2.5 and Lemma 2.6, we have

$$
\left\|\mathcal{T}_{u^{0}}(u)-S_{\alpha}(t) u^{0}\right\|_{X}=\|T(g(u))\|_{X} \leq C_{1}\|g(u)\|_{Y} \leq C_{1}[u]_{X}^{2} \leq C_{1} C_{2}^{2}(\rho+\varepsilon)^{2} .
$$

Therefore $\mathcal{T}_{u^{0}}$ maps $\mathcal{B}_{\rho}\left(u^{0}\right)$ into itself provided that

$$
C_{1} C_{2}^{2}(\rho+\varepsilon)^{2} \leq \rho .
$$

Notice that by (2.14), the condition (2.27) implies that (2.31) is satisfied.

To prove (2.28), we use the decomposition

$$
\left.g(u)-g(v)=-2 i(\beta-i \alpha)\left[\left(\frac{\bar{u}}{1+|u|^{2}}-\frac{\bar{v}}{1+|v|^{2}}\right)(\nabla u)^{2}+\frac{\bar{v}}{1+|v|^{2}}\left((\nabla u)^{2}-(\nabla v)^{2}\right)\right)\right] .
$$

Since

$$
\left|\frac{\bar{u}}{1+|u|^{2}}-\frac{\bar{v}}{1+|v|^{2}}\right| \leq|u-v| \frac{1+|u||v|}{\left(1+|u|^{2}\right)\left(1+|v|^{2}\right)} \leq|u-v|
$$

and using (2.2), we obtain

$$
|g(u)-g(v)| \leq 2|u-v||\nabla u|^{2}+|\nabla u-\nabla v|(|\nabla u|+|\nabla v|) .
$$

Therefore

$$
\|g(u)-g(v)\|_{Y} \leq 2\left\|\left|u-v\left\||\nabla u|^{2}\right\|_{Y}+\||\nabla u-\nabla v|(|\nabla u|+|\nabla v|)\|_{Y}:=I_{1}+I_{2} .\right.\right.
$$

For $I_{1}$, it is immediate that

$$
I_{1} \leq 2 \sup _{t>0}\|u-v\|_{L^{\infty}}\left[\left(\sup _{t>0} \sqrt{t}\|\nabla u\|_{L^{\infty}}\right)^{2}+\sup _{\substack{x \in \mathbb{R}^{N} \\ r>0}} \frac{1}{r^{N}} \int_{Q_{r}(x)}|\nabla u|^{2} d t d y\right] \leq 2\|u-v\|_{X}[u]_{X}^{2} .
$$


Similarly, using the Cauchy-Schwarz inequality,

$$
\begin{aligned}
I_{2} \leq & \left(\sup _{t>0} \sqrt{t}\|\nabla u-\nabla v\|_{L^{\infty}}\right)\left(\sup _{t>0} \sqrt{t}\left(\|\nabla u\|_{L^{\infty}}+\|\nabla v\|_{L^{\infty}}\right)\right) \\
& +\sup _{\substack{x \in \mathbb{R}^{N} \\
r>0}} \frac{1}{r^{N}}\left(\|\nabla u-\nabla v\|_{L^{2}\left(Q_{r}(x)\right)}\right)\left(\|\nabla u\|_{L^{2}\left(Q_{r}(x)\right)}+\|\nabla v\|_{L^{2}\left(Q_{r}(x)\right)}\right) \\
\leq & \|u-v\|_{X}\left([u]_{X}+[v]_{X}\right) .
\end{aligned}
$$

Using Lemma 2.4, (2.32), (2.33) and (2.34), we conclude that

$$
\|T(g(u))-T(g(v))\|_{X} \leq C_{1}\left(2[u]_{X}^{2}+[u]_{X}+[v]_{X}\right)\|u-v\|_{X} .
$$

Let $u, v \in \mathcal{B}_{\rho}\left(u^{0}\right)$, by Lemma 2.5 and $(2.30)$

$$
[u]_{X} \leq C_{2}(\rho+\varepsilon) \leq \frac{5}{32}
$$

so that

$$
2[u]_{X}^{2}+[u]_{X}+[v]_{X} \leq \frac{37}{16} C_{2}(\rho+\varepsilon)<3 C_{2}(\rho+\varepsilon) .
$$

Then (2.35) implies that

$$
\left\|\mathcal{T}_{u^{0}}(u)-\mathcal{T}_{u^{0}}(v)\right\|_{X} \leq 3 C_{1} C_{2}(\rho+\varepsilon)\|u-v\|_{X} .
$$

From (2.29), we conclude that

$$
3 C_{1} C_{2}(\rho+\varepsilon) \leq \frac{15}{32} \leq \frac{1}{2},
$$

and then (2.38) yields that the operator $\mathcal{T}_{u^{0}}$ defined in $(2.17)$ is a contraction on $\mathcal{B}_{\rho}\left(u^{0}\right)$. This concludes the proof of the proposition.

Proof of Theorem 2.1. Let us set $C=C_{1} C_{2}^{2}$ and $K=C_{2}$, where $C_{1}$ and $C_{2}$ are the constants in Lemma 2.4 and Lemma 2.5 respectively. Since $\rho$ satisfies (2.6), Proposition 2.7 implies that there exists a solution $u$ of equation $(2.16)$ in the ball $\mathcal{B}_{\rho}\left(u^{0}\right)$, and in particular from Lemma 2.5

$$
\sup _{t>0}\|u\|_{L^{\infty}} \leq K(\rho+L) \quad \text { and } \quad[u]_{X} \leq K(\rho+\varepsilon) .
$$

To prove the uniqueness part of the theorem, let us assume that $u$ and $v$ are solutions of (IDNLS) in $X\left(\mathbb{R}^{N} \times \mathbb{R}^{+} ; \mathbb{C}\right)$ such that

$$
[u]_{X},[v]_{X} \leq K(\rho+\varepsilon),
$$

with the same initial condition $u^{0}$. By the definitions of $C$ and $K,(2.6)$ and (2.40), the estimates in (2.29) and (2.30) hold. It follows that (2.36), (2.37) and (2.39) are satisfied. Then, using $(2.28)$

$$
\begin{aligned}
\|u-v\|_{X} & =\|T(g(u))-T(g(v))\|_{X} \leq C_{1}\left(2[u]_{X}^{2}+[u]_{X}+[v]_{X}\right)\|u-v\|_{X} \\
& \leq \frac{1}{2}\|u-v\|_{X} .
\end{aligned}
$$

From which it follows that $u=v$.

To prove the dependence of the solution with respect to the initial data (part (iv)), consider $u$ and $v$ solutions of (IDNLS) satisfying (2.40) with initial conditions $u^{0}$ and $v^{0}$. Then, by definition, $u=\mathcal{T}_{u^{0}}(u), v=\mathcal{T}_{v^{0}}(v)$ and

$$
\|u-v\|_{X}=\left\|\mathcal{T}_{u^{0}}(u)-\mathcal{T}_{v^{0}}(v)\right\|_{X} \leq\left\|S_{\alpha}\left(u^{0}-v^{0}\right)\right\|_{X}+\|T(g(u))-T(g(v))\|_{X} .
$$


Using (2.15), (2.23) and (2.28) and arguing as above, we have

$$
\begin{aligned}
\|u-v\|_{X} & \leq C_{2}\left(\left\|u^{0}-v^{0}\right\|_{L^{\infty}}+\left[u^{0}-v^{0}\right]_{B M O}\right)+C_{1}\left(2[u]_{X}^{2}+[u]_{X}+[v]_{X}\right)\|u-v\|_{X} \\
& \leq 3 C_{2}\left\|u^{0}-v^{0}\right\|_{L^{\infty}}+\frac{1}{2}\|u-v\|_{X} .
\end{aligned}
$$

This yields (2.10), since $K=C_{2}$.

The assertions in (ii) and (iii) follow from Theorem A.3.

\subsection{The Cauchy problem for the LLG equation}

By using the inverse of the stereographic projection $\mathcal{P}^{-1}: \mathbb{C} \rightarrow \mathbb{S}^{2} \backslash\{0,0,-1\}$, that is explicitly given by $\boldsymbol{m}=\left(m_{1}, m_{2}, m_{3}\right)=\mathcal{P}^{-1}(u)$, with

$$
m_{1}=\frac{2 \operatorname{Re} u}{1+|u|^{2}}, \quad m_{2}=\frac{2 \operatorname{Im} u}{1+|u|^{2}}, \quad m_{3}=\frac{1-|u|^{2}}{1+|u|^{2}},
$$

we will be able to establish the following global well-posedness result for $\left(\mathrm{LLG}_{\alpha}\right)$.

Theorem 2.9. Let $\alpha \in(0,1]$. There exist constants $C \geq 1$ and $K \geq 4$, such that for any $\delta \in(0,2], \varepsilon_{0}>0$ and $\rho>0$ such that

$$
8 K^{4} C \delta^{-4}\left(\rho+8 \delta^{-2} \varepsilon_{0}\right)^{2} \leq \rho,
$$

if $\boldsymbol{m}^{0}=\left(m_{1}^{0}, m_{2}^{0}, m_{3}^{0}\right) \in L^{\infty}\left(\mathbb{R}^{N} ; \mathbb{S}^{2}\right)$ satisfies

$$
\inf _{\mathbb{R}^{N}} m_{3}^{0} \geq-1+\delta \quad \text { and } \quad\left[\boldsymbol{m}^{0}\right]_{B M O} \leq \varepsilon_{0},
$$

then there exists a unique solution $\boldsymbol{m}=\left(m_{1}, m_{2}, m_{3}\right) \in X\left(\mathbb{R}^{N} \times \mathbb{R}^{+} ; \mathbb{S}^{2}\right)$ of $\left(\mathrm{LLG}_{\alpha}\right)$ such that

$$
\inf _{\substack{x \in \mathbb{R}^{N} \\ t>0}} m_{3}(x, t) \geq-1+\frac{2}{1+K^{2}\left(\rho+\delta^{-1}\right)^{2}} \quad \text { and } \quad[\boldsymbol{m}]_{X} \leq 4 K\left(\rho+8 \delta^{-2} \varepsilon_{0}\right) .
$$

Moreover, we have the following properties.

i) $\boldsymbol{m} \in C^{\infty}\left(\mathbb{R}^{N} \times \mathbb{R}^{+} ; \mathbb{S}^{2}\right)$.

ii) $\left|\boldsymbol{m}(\cdot, t)-\boldsymbol{m}^{0}\right| \longrightarrow 0$ in $S^{\prime}\left(\mathbb{R}^{N}\right)$ as $t \longrightarrow 0^{+}$.

iii) Assume that $\boldsymbol{m}$ and $\boldsymbol{n}$ are respectively smooth solutions to (IDNLS) satisfying (2.44) with initial conditions $\boldsymbol{m}^{0}$ and $\boldsymbol{n}^{0}$ satisfying (2.43). Then

$$
\|\boldsymbol{m}-\boldsymbol{n}\|_{X} \leq 120 K \delta^{-2}\left\|\boldsymbol{m}^{0}-\boldsymbol{n}^{0}\right\|_{L^{\infty}} .
$$

Remark 2.10. The restriction (2.42) on the parameters is similar to (2.27), but we need to include $\delta$. To better understand the role of $\delta$, we can proceed as before. Indeed, setting for $a, \delta>0$,

$$
\mathcal{S}_{\delta}(a)=\left\{\left(\rho, \varepsilon_{0}\right) \in \mathbb{R}^{+} \times \mathbb{R}^{+}: a \delta^{-4}\left(\rho+8 \delta^{-2} \varepsilon_{0}\right)^{2} \leq \rho\right\},
$$

we see that its shape is similar to the one in Figure 1. It is simple to verify that for any $\left(\rho, \varepsilon_{0}\right) \in \mathcal{S}_{\delta}(a)$, we have the bounds

$$
\rho \leq \frac{\delta^{4}}{a} \quad \text { and } \quad \varepsilon_{0} \leq \frac{\delta^{6}}{32 a},
$$

and the maximum value $\varepsilon_{0}^{*}=\frac{\delta^{6}}{32 a}$ is attained at $\rho^{*}=\frac{\delta^{4}}{4 a}$. Also, the sets are well ordered, i.e. if $\tilde{a} \geq a>0$, then $\mathcal{S}_{\delta}(\tilde{a}) \subseteq \mathcal{S}_{\delta}(a)$. 
We emphasize that the first condition in (2.43) is rather technical. Indeed, we need the essential range of $\boldsymbol{m}^{0}$ to be far from the South Pole in order to use the stereographic projection. In the case $\alpha=1$, Wang [35] proved the global well-posedness using only the second restriction in (2.43). It is an open problem to determinate if this condition is necessary in the case $\alpha \in(0,1)$.

The choice of the South Pole is of course arbitrary. By using the invariance of (LLG $\left.\mathrm{L}_{\alpha}\right)$ under rotations, we have the existence of solutions provided that the essential range of the initial condition $\boldsymbol{m}^{0}$ is far from an arbitrary point $\boldsymbol{Q} \in \mathbb{S}^{2}$. Precisely,

Corollary 2.11. Let $\alpha \in(0,1], \boldsymbol{Q} \in \mathbb{S}^{2}, \delta \in(0,2]$, and $\varepsilon_{0}, \rho>0$ such that (2.42) holds. Given $\boldsymbol{m}^{0}=\left(m_{1}^{0}, m_{2}^{0}, m_{3}^{0}\right) \in L^{\infty}\left(\mathbb{R}^{N} ; \mathbb{S}^{2}\right)$ satisfying

$$
\inf _{\mathbb{R}^{N}}\left|\boldsymbol{m}^{0}-\boldsymbol{Q}\right|^{2} \geq 2 \delta \quad \text { and } \quad\left[\boldsymbol{m}^{0}\right]_{B M O} \leq \varepsilon_{0},
$$

there exists a unique smooth solution $\boldsymbol{m} \in X\left(\mathbb{R}^{N} \times \mathbb{R}^{+} ; \mathbb{S}^{2}\right)$ of $\left(\mathrm{LLG}_{\alpha}\right)$ with initial condition $\boldsymbol{m}^{0}$ such that

$$
\inf _{\substack{x \in \mathbb{R}^{N} \\ t>0}}|\boldsymbol{m}(x, t)-\boldsymbol{Q}|^{2} \geq \frac{4}{1+K^{2}\left(\rho+\delta^{-1}\right)^{2}} \quad \text { and } \quad[\boldsymbol{m}]_{X} \leq 4 K\left(\rho+8 \delta^{-2} \varepsilon_{0}\right) .
$$

For the sake of clarity, before proving Theorem 2.9, we provide a precise meaning of what we refer to as a weak and smooth global solution of the $\left(L_{L} G_{\alpha}\right)$ equation. The definition below is motivated by the following vector identities for a smooth function $\boldsymbol{m}$ with $|\boldsymbol{m}|=1$ :

$$
\begin{gathered}
\boldsymbol{m} \times \Delta \boldsymbol{m}=\operatorname{div}(\boldsymbol{m} \times \nabla \boldsymbol{m}), \\
-\boldsymbol{m} \times(\boldsymbol{m} \times \Delta \boldsymbol{m})=\Delta \boldsymbol{m}+|\nabla \boldsymbol{m}|^{2} \boldsymbol{m} .
\end{gathered}
$$

Definition 2.12. Let $T \in(0, \infty]$ and $\boldsymbol{m}^{0} \in L^{\infty}\left(\mathbb{R}^{N} ; \mathbb{S}^{2}\right)$. We say that

$$
\boldsymbol{m} \in L_{\mathrm{loc}}^{\infty}\left((0, T), H_{\mathrm{loc}}^{1}\left(\mathbb{R}^{N} ; \mathbb{S}^{2}\right)\right)
$$

is a weak solution of $\left(\mathrm{LLG}_{\alpha}\right)$ in $(0, T)$ with initial condition $\boldsymbol{m}^{0}$ if

$$
-\left\langle\boldsymbol{m}, \partial_{t} \varphi\right\rangle=\beta\langle\boldsymbol{m} \times \nabla \boldsymbol{m}, \nabla \varphi\rangle-\alpha\langle\nabla \boldsymbol{m}, \nabla \varphi\rangle+\alpha\left\langle|\nabla \boldsymbol{m}|^{2} \boldsymbol{m}, \varphi\right\rangle,
$$

and

$$
\left\|\left(\boldsymbol{m}(t)-\boldsymbol{m}^{0}\right) \varphi\right\|_{L^{1}} \rightarrow 0, \quad \text { as } t \rightarrow 0^{+}, \quad \text { for all } \varphi \in C_{0}^{\infty}\left(\mathbb{R}^{N} \times(0, T)\right) .
$$

If $T=\infty$, and in addition $\boldsymbol{m} \in C^{\infty}\left(\mathbb{R}^{N} \times \mathbb{R}^{+}\right)$, we say that $\boldsymbol{m}$ is a smooth global solution of $\left(\mathrm{LLG}_{\alpha}\right)$ in $\mathbb{R}^{N} \times \mathbb{R}^{+}$with initial condition $\boldsymbol{m}^{0}$. Here $\langle\cdot, \cdot\rangle$ stands for

$$
\left\langle\boldsymbol{f}_{1}, \boldsymbol{f}_{2}\right\rangle=\int_{0}^{\infty} \int_{\mathbb{R}^{N}} \boldsymbol{f}_{1} \cdot \boldsymbol{f}_{2} d x d t .
$$

With this definition, we see the following: Assume that $\boldsymbol{m}$ is a smooth global solution of $\left(\mathrm{LLG}_{\alpha}\right)$ with initial condition $\boldsymbol{m}^{0}$ and consider its stereographic projection $\mathcal{P}(\boldsymbol{m})$. If $\mathcal{P}(\boldsymbol{m})$ and $\mathcal{P}\left(\boldsymbol{m}^{0}\right)$ are well-defined, then $\mathcal{P}(\boldsymbol{m}) \in C^{\infty}\left(\mathbb{R}^{N} \times \mathbb{R}^{+} ; \mathbb{C}\right.$ ) satisfies (DNLS) pointwise, and

$$
\lim _{t \rightarrow 0^{+}} \mathcal{P}(\boldsymbol{m})=\mathcal{P}\left(\boldsymbol{m}^{0}\right) \quad \text { in } S^{\prime}\left(\mathbb{R}^{N}\right) .
$$

Therefore, if in addition $\mathcal{P}(\boldsymbol{m}) \in X\left(\mathbb{R}^{N} \times \mathbb{R} ; \mathbb{C}\right)$, then $\mathcal{P}(\boldsymbol{m})$ is a smooth global solution of (DNLS) with initial condition $\mathcal{P}\left(\boldsymbol{m}^{0}\right)$. Reciprocally, suppose that $u \in X\left(\mathbb{R}^{N} \times \mathbb{R}^{+} ; \mathbb{C}\right) \cap C^{\infty}\left(\mathbb{R}^{N} \times \mathbb{R}^{+}\right)$ is a solution of (IDNLS) with initial condition $u^{0} \in L^{\infty}\left(\mathbb{R}^{N}\right)$ such that $(2.9)$ holds. If $\mathcal{P}^{-1}(u)$ and $\mathcal{P}^{-1}\left(u^{0}\right)$ are in appropriate spaces, then $\mathcal{P}^{-1}(u)$ is a global smooth solution of (LLG $\left.{ }_{\alpha}\right)$ with initial condition $\mathcal{P}^{-1}\left(u^{0}\right)$. The above (formal) argument allows us to obtain Theorem 2.9 from Theorem 2.1 once we have established good estimates for the mappings $\mathcal{P}$ and $\mathcal{P}^{-1}$. In this context, we have the following 
Lemma 2.13. Let $u, v \in C^{1}\left(\mathbb{R}^{N} ; \mathbb{C}\right), \boldsymbol{m}=\left(m_{1}, m_{2}, m_{3}\right), \boldsymbol{n}=\left(n_{1}, n_{2}, n_{3}\right) \in C^{1}\left(\mathbb{R}^{N} ; \mathbb{S}^{2}\right)$.

a) Assume that $\inf _{\mathbb{R}^{N}} m_{3} \geq-1+\delta$ and $\inf _{\mathbb{R}^{N}} n_{3} \geq-1+\delta$ for some constant $\delta \in(0,2]$. If $u=\mathcal{P}(\boldsymbol{m})$ and $v=\mathcal{P}(\boldsymbol{n})$, then

$$
\begin{aligned}
|u(x)-v(x)| & \leq \frac{4}{\delta^{2}}|\boldsymbol{m}(x)-\boldsymbol{n}(x)|, \\
{[u]_{B M O} } & \leq \frac{8}{\delta^{2}}[\boldsymbol{m}]_{B M O}, \\
|\nabla u(x)| & \leq \frac{4}{\delta^{2}}|\nabla \boldsymbol{m}(x)|,
\end{aligned}
$$

for all $x \in \mathbb{R}^{N}$.

b) Assume that $\|u\|_{L^{\infty}} \leq M$, $\|v\|_{L^{\infty}} \leq M$, for some constant $M \geq 0$. If $\boldsymbol{m}=\mathcal{P}^{-1}(u)$ and $\boldsymbol{n}=\mathcal{P}^{-1}(v)$, then

$$
\begin{aligned}
\inf _{\mathbb{R}^{N}} m_{3} & \geq-1+\frac{2}{1+M^{2}}, \\
|\boldsymbol{m}(x)-\boldsymbol{n}(x)| & \leq 3|u(x)-v(x)|, \\
|\nabla \boldsymbol{m}(x)| & \leq 4|\nabla u(x)|, \\
|\nabla \boldsymbol{m}(x)-\nabla \boldsymbol{n}(x)| & \leq 4|\nabla u(x)-\nabla v(x)|+12|u(x)-v(x)|(|\nabla u(x)|+|\nabla v(x)|) .
\end{aligned}
$$

Proof. In the proof we will use the notation $\check{m}:=m_{1}+i m_{2}$. To establish (2.49), we write

$$
u(x)-v(x)=\frac{\check{m}(x)-\check{n}(x)}{1+m_{3}(x)}+\frac{\check{n}(x)\left(n_{3}(x)-m_{3}(x)\right)}{\left(1+m_{3}(x)\right)\left(1+n_{3}(x)\right)} .
$$

Hence, since $|\check{n}| \leq 1, m_{3}(x)+1 \geq \delta$ and $n_{3}(x)+1 \geq \delta, \forall x \in \mathbb{R}^{N}$,

$$
|u(x)-v(x)| \leq \frac{|\check{m}(x)-\check{n}(x)|}{\delta}+\frac{\left|n_{3}(x)-m_{3}(x)\right|}{\delta^{2}} .
$$

Using that

$$
|\check{m}-\check{n}| \leq|\boldsymbol{m}-\boldsymbol{n}|
$$

and that

$$
\max \left\{\frac{1}{a}, \frac{1}{a^{2}}\right\} \leq \frac{2}{a^{2}}, \text { for all } a \in(0,2],
$$

we obtain (2.49). The same argument also shows that

$$
|u(y)-u(z)| \leq \frac{4}{\delta^{2}}|\boldsymbol{m}(y)-\boldsymbol{m}(z)|, \quad \text { for all } y, z \in \mathbb{R}^{N} .
$$

To verify (2.50), we recall the following inequalities in BMO (see [10]):

$$
[f]_{B M O} \leq \sup _{x \in \mathbb{R}^{N}} f_{B_{r}(x)} f_{B_{r}(x)}|f(y)-f(z)| d y d z \leq 2[f]_{B M O} .
$$

Estimate (2.50) is an immediate consequence of this inequality and (2.57). To prove (2.51) it is enough to remark that

$$
|\nabla u| \leq \frac{2}{\delta^{2}}\left(\left|\nabla m_{1}\right|+\left|\nabla m_{2}\right|+\left|\nabla m_{3}\right|\right) \leq \frac{4}{\delta^{2}}|\nabla \boldsymbol{m}| .
$$


We turn into (b). Using the explicit formula for $\mathcal{P}^{-1}$ in $(2.41)$, we can write

$$
m_{3}=-1+\frac{2}{1+|u|^{2}} .
$$

Since $\|u\|_{L^{\infty}} \leq M$, we obtain (2.52).

To show (2.53), we compute

$$
\begin{aligned}
\check{m}-\check{n} & =\frac{2 u}{1+|u|^{2}}-\frac{2 v}{1+|v|^{2}}=\frac{2(u-v)+2 u v(\bar{v}-\bar{u})}{\left(1+|u|^{2}\right)\left(1+|v|^{2}\right)}, \\
m_{3}-n_{3} & =\frac{1-|u|^{2}}{1+|u|^{2}}-\frac{1-|v|^{2}}{1+|v|^{2}}=\frac{2\left(|v|^{2}-|u|^{2}\right)}{\left(1+|u|^{2}\right)\left(1+|v|^{2}\right)} .
\end{aligned}
$$

Using the inequalities

$$
\frac{a}{1+a^{2}} \leq \frac{1}{2}, \quad \frac{1+a b}{\left(1+a^{2}\right)\left(1+b^{2}\right)} \leq 1, \quad \text { and } \quad \frac{a+b}{\left(1+a^{2}\right)\left(1+b^{2}\right)} \leq 1, \quad \text { for all } a, b \geq 0,
$$

from (2.59) and (2.60) we deduce that

$$
|\check{m}-\check{n}| \leq 2|u-v| \quad \text { and } \quad\left|m_{3}-n_{3}\right| \leq 2|u-v| .
$$

Hence

$$
|\boldsymbol{m}-\boldsymbol{n}|=\sqrt{|\check{m}-\check{n}|^{2}+\left|m_{3}-n_{3}\right|^{2}} \leq \sqrt{8}|u-v| \leq 3|u-v| .
$$

To estimate the gradient, we compute

$$
\nabla \check{m}=\frac{2 \nabla u}{1+|u|^{2}}-\frac{4 u \operatorname{Re}(\bar{u} \nabla u)}{\left(1+|u|^{2}\right)^{2}},
$$

from which it follows that

$$
|\nabla \check{m}| \leq|\nabla u|\left(\frac{2}{1+|u|^{2}}+\frac{4|u|^{2}}{\left(1+|u|^{2}\right)^{2}}\right) \leq 3|\nabla u|,
$$

since $\frac{4 a}{(1+a)^{2}} \leq 1$, for all $a \geq 0$. For $m_{3}$, we have

$$
\nabla m_{3}=-\frac{2 \operatorname{Re}(\bar{u} \nabla u)}{1+|u|^{2}}-\frac{2 \operatorname{Re}(\bar{u} \nabla u)\left(1-|u|^{2}\right)}{\left(1+|u|^{2}\right)^{2}}=-\frac{4 \operatorname{Re}(\bar{u} \nabla u)}{\left(1+|u|^{2}\right)^{2}},
$$

and therefore $\left|\nabla m_{3}\right| \leq 2|\nabla u|$, since

$$
\frac{a}{\left(1+a^{2}\right)^{2}} \leq \frac{1}{2}, \quad \text { for all } a \geq 0 .
$$

Hence

$$
|\nabla \boldsymbol{m}|=\sqrt{\left|\nabla m_{1}\right|^{2}+\left|\nabla m_{2}\right|^{2}+\left|\nabla m_{3}\right|^{2}} \leq \sqrt{13}|\nabla u| \leq 4|\nabla u|,
$$

which gives (2.54).

In order to prove (2.55), we start differentiating (2.59)

$$
\begin{aligned}
\nabla \check{m}-\nabla \check{n}= & 2 \frac{\nabla(u-v)+\nabla(u v)(\bar{v}-\bar{u})+u v \nabla(\bar{v}-\bar{u})}{\left(1+|u|^{2}\right)\left(1+|v|^{2}\right)} \\
& -4 \frac{((u-v)+u v(\bar{v}-\bar{u}))\left(\operatorname{Re}(\bar{u} \nabla u)\left(1+|v|^{2}\right)+\operatorname{Re}(\bar{v} \nabla v)\left(1+|u|^{2}\right)\right)}{\left(1+|u|^{2}\right)^{2}\left(1+|v|^{2}\right)^{2}},
\end{aligned}
$$


Hence, setting $R=\max \{|\nabla u(x)|,|\nabla v(x)|\}$,

$$
\begin{aligned}
|\nabla \check{m}-\nabla \check{n}| \leq & 2|\nabla u-\nabla v|\left(\frac{1+|u||v|}{\left(1+|u|^{2}\right)\left(1+|v|^{2}\right)}\right)+2 R|u-v|\left(\frac{|u|+|v|}{\left(1+|u|^{2}\right)\left(1+|v|^{2}\right)}\right) \\
& +4 R|u-v|\left(\frac{|u|(1+|u||v|)}{\left(1+|u|^{2}\right)^{2}\left(1+|v|^{2}\right)}+\frac{|v|(1+|u||v|)}{\left(1+|u|^{2}\right)\left(1+|v|^{2}\right)^{2}}\right) .
\end{aligned}
$$

Using again (2.61), we get

$$
\frac{|u|(1+|u||v|)}{\left(1+|u|^{2}\right)^{2}\left(1+|v|^{2}\right)} \leq \frac{|u|}{\left(1+|u|^{2}\right)} \leq \frac{1}{2}
$$

By symmetry, the same estimate holds interchanging $u$ by $v$. Therefore, invoking again (2.61), we obtain

$$
|\nabla \check{m}-\nabla \check{n}| \leq 2|\nabla u-\nabla v|+6 R|u-v| .
$$

Similarly, writing $|u|^{2}-|v|^{2}=(u-v) \bar{u}+(\bar{u}-\bar{v}) v$, from (2.60) we have

$$
\left|\nabla m_{3}-\nabla n_{3}\right| \leq 2|\nabla u-\nabla v|+6 R|u-v| .
$$

Therefore, since

$$
\sqrt{a^{2}+b^{2}} \leq a+b, \quad \forall a, b \geq 0,
$$

inequalities (2.65) and (2.66) yield (2.55).

Now we have all the elements to establish Theorem 2.9.

Proof of Theorem 2.9. We continue to use the constants $C$ and $K$ defined in Theorem 2.1. We recall that they are given by $C=C_{1} C_{2}^{2}$ and $K=C_{2}$, where $C_{1} \geq 1$ and $C_{2} \geq 1$ are the constants in Lemmas 2.4 and 2.5, respectively. In addition, w.l.o.g. we assume that

$$
K=C_{2} \geq 4
$$

in order to simplify our computations.

First we notice that by Remark 2.10, any $\rho$ and $\varepsilon_{0}$ fulfilling the condition (2.42), also satisfy

$$
8 C\left(\rho+8 \delta^{-2} \varepsilon_{0}\right)^{2} \leq \rho,
$$

since $\delta^{4} / K^{4} \leq 1$ (notice that $K \geq 4$ and $\delta \in(0,2]$ ).

Let $\boldsymbol{m}^{0}$ as in the statement of the theorem and set $u^{0}=\mathcal{P}\left(\boldsymbol{m}^{0}\right)$. Using (2.50) in Lemma 2.13, we have

$$
\left\|u^{0}\right\|_{L^{\infty}} \leq\left\|\frac{1}{1+m_{3}^{0}}\right\|_{L^{\infty}} \leq \frac{1}{\delta} \quad \text { and } \quad\left[u^{0}\right]_{B M O} \leq \frac{8 \varepsilon_{0}}{\delta^{2}} .
$$

Therefore, bearing in mind (2.68), we can apply Theorem 2.1 with

$$
L:=\frac{1}{\delta} \quad \text { and } \quad \varepsilon:=8 \delta^{-2} \varepsilon_{0},
$$

to obtain a smooth solution $u \in X\left(\mathbb{R}^{N} \times \mathbb{R}^{+} ; \mathbb{C}\right)$ to (IDNLS) with initial condition $u^{0}$. In particular $u$ satisfies

$$
\sup _{t>0}\|u\|_{L^{\infty}} \leq K\left(\rho+\delta^{-1}\right) \quad \text { and } \quad[u]_{X} \leq K\left(\rho+8 \delta^{-2} \varepsilon_{0}\right) .
$$


Defining $\boldsymbol{m}=\mathcal{P}^{-1}(u)$, we infer that $\boldsymbol{m}$ is a smooth solution to $\left(\mathrm{LLG}_{\alpha}\right)$ and, using the fact that $\left\|\left(u(\cdot, t)-u^{0}\right) \varphi\right\|_{L^{1}} \rightarrow 0$ (see $\left.(2.9)\right)$ and $(2.53)$,

$$
\left|\boldsymbol{m}(\cdot, t)-\boldsymbol{m}^{0}\right| \longrightarrow 0 \quad \text { in } \mathcal{S}^{\prime}\left(\mathbb{R}^{N}\right), \text { as } t \rightarrow 0^{+} .
$$

Notice also that applying Lemma 2.13 we obtain

$$
\inf _{\substack{x \in \mathbb{R}^{N} \\ t>0}} m_{3}(x, t) \geq-1+\frac{2}{1+K^{2}\left(\rho+\delta^{-1}\right)^{2}} \quad \text { and } \quad[\boldsymbol{m}]_{X} \leq 4[u]_{X} \leq 4 K\left(\rho+8 \delta^{-2} \varepsilon_{0}\right),
$$

which yields (2.44).

Let us now prove the uniqueness. Let $\boldsymbol{n}$ be a another smooth solution of (LLG $\left.\mathrm{L}_{\alpha}\right)$ with initial condition $u^{0}$ satisfying

$$
\inf _{\substack{x \in \mathbb{R}^{N} \\ t>0}} n_{3}(x, t) \geq-1+\frac{2}{1+K^{2}\left(\rho+\delta^{-1}\right)^{2}} \quad \text { and } \quad[\boldsymbol{n}]_{X} \leq 4 K\left(\rho+8 \delta^{-2} \varepsilon_{0}\right),
$$

and let $v=\mathcal{P}(\boldsymbol{n})$ be its stereographic projection. Then by (2.51),

$$
[v]_{X} \leq\left(1+K^{2}\left(\rho+\delta^{-1}\right)^{2}\right)^{2}[\boldsymbol{n}]_{X} .
$$

We continue to control the upper bounds for $[v]_{X}$ and $[u]_{X}$ in terms of $\delta$ and the constants $C_{1} \geq 1$ and $C_{2} \geq 4$. Notice that since $\rho$ and $\varepsilon_{0}$ satisfy (2.42), from (2.46) with $a=8 K^{4} C$, it follows that

$$
\rho \leq \frac{\delta^{4}}{8 K^{4} C} \quad \text { and } \quad \varepsilon_{0} \leq \frac{\delta^{6}}{2^{8} K^{4} C}
$$

or equivalently (recall that $K=C_{2}$ and $C=C_{1} C_{2}^{2}$ )

$$
\rho \leq \frac{\delta^{4}}{8 C_{1} C_{2}^{6}} \quad \text { and } \quad \frac{8 \varepsilon_{0}}{\delta^{2}} \leq \frac{\delta^{4}}{32 C_{1} C_{2}^{6}} .
$$

Hence

$$
K\left(\rho+8 \delta^{-2} \varepsilon_{0}\right) \leq \frac{5 \delta^{4}}{32 C_{1} C_{2}^{5}}
$$

Also, using (2.72), we have

$$
\begin{aligned}
1+K^{2}\left(\rho+\delta^{-1}\right)^{2} & =1+\frac{C_{2}^{2}}{\delta^{2}}(\rho \delta+1)^{2}=\frac{C_{2}^{2}}{\delta^{2}}\left(\frac{\delta^{2}}{C_{2}^{2}}+(\rho \delta+1)^{2}\right) \\
& \leq \frac{C_{2}^{2}}{\delta^{2}}\left(\frac{\delta^{2}}{C_{2}^{2}}+\left(\frac{\delta^{5}}{8 C_{1} C_{2}^{6}}+1\right)^{2}\right) \leq 2 \frac{C_{2}^{2}}{\delta^{2}}
\end{aligned}
$$

since $C_{1} \geq 1, C_{2} \geq 4$ and $\delta \leq 2$.

From the bounds in (2.73) and (2.74), combined with (2.69), (2.70) and (2.71), we obtain

$$
[u]_{X} \leq K\left(\rho+8 \delta^{-2} \varepsilon_{0}\right) \leq \frac{5 \delta^{4}}{32 C_{1} C_{2}^{5}} \leq \frac{5}{2^{11} C_{1}}
$$

and

$$
[v]_{X} \leq\left(1+K^{2}\left(\rho+\delta^{-1}\right)^{2}\right)^{2}[\boldsymbol{n}]_{X} \leq\left(1+K^{2}\left(\rho+\delta^{-1}\right)^{2}\right)^{2} 4 K\left(\rho+8 \delta^{-2} \varepsilon_{0}\right) \leq\left(2 \frac{C_{2}^{2}}{\delta^{2}}\right)^{2} \frac{20 \delta^{4}}{32 C_{1} C_{2}^{5}} \leq \frac{5}{8 C_{1}}
$$


since $\delta \leq 2$ and $C_{2} \geq 4$. Finally, since $u$ and $v$ are solutions to (IDNLS) with initial condition $u^{0},(2.28)$ and the above inequalities for $[u]_{X}$ and $[v]_{X}$ yield

$$
\begin{aligned}
\|u-v\|_{X} & \leq C_{1}\left(2[u]_{X}^{2}+[u]_{X}+[v]_{X}\right)\|u-v\|_{X} \\
& \leq C_{1}\left(2\left(\frac{5}{2^{11} C_{1}}\right)^{2}+\frac{5}{2^{11} C_{1}}+\frac{5}{8 C_{1}}\right)\|u-v\|_{X},
\end{aligned}
$$

which implies that $u=v$, bearing in mind that the constant on the r.h.s. of the above inequality is strictly less that one. This completes the proof of the uniqueness.

It remains to establish (2.45). Let $\boldsymbol{m}$ and $\boldsymbol{n}$ two smooth solutions of (LLG ${ }_{\alpha}$ ) satisfying (2.44). As a consequence of the uniqueness, we see that $\boldsymbol{m}$ and $\boldsymbol{n}$ are the inverse stereographic projection of some functions $u$ and $v$ that are solutions of (IDNLS) with initial condition $u^{0}=\mathcal{P}\left(\boldsymbol{m}^{0}\right)$ and $v^{0}=\mathcal{P}\left(\boldsymbol{n}^{0}\right)$, respectively. In particular, $u$ and $v$ satisfy the estimates in (2.69). Using also (2.53) and (2.55), we deduce that

$$
\begin{aligned}
\|\boldsymbol{m}-\boldsymbol{n}\|_{X} & \left.\leq 3 \sup _{t>0}\|u-v\|_{L^{\infty}}+4[u-v]_{X}+12 \sup _{t>0}\|u-v\|_{L^{\infty}}\left([u]_{X}+[v]_{X}\right]\right) \\
& \leq 4\|u-v\|_{X}+24 C_{2}\left(\rho+8 \delta^{-2} \varepsilon_{0}\right)\|u-v\|_{X} \\
& \leq 5\|u-v\|_{X}
\end{aligned}
$$

where we have used (2.73) in obtaining the last inequality. Finally, using also (2.43) and (2.49), and applying (2.10) in Theorem 2.1,

$$
\begin{aligned}
\|\boldsymbol{m}-\boldsymbol{n}\|_{X} & \leq 30 K\left\|u^{0}-v^{0}\right\|_{L^{\infty}} \\
& \leq 120 K \delta^{-2}\left\|\boldsymbol{m}^{0}-\boldsymbol{n}^{0}\right\|_{L^{\infty}}
\end{aligned}
$$

which yields (2.45).

Proof of Corollary 2.11. Let $\mathcal{R} \in S O(3)$ such that $\mathcal{R} \boldsymbol{Q}=(0,0,-1)$, i.e. $\mathcal{R}$ is the rotation that maps $\boldsymbol{Q}$ to the South Pole. Let us set $\boldsymbol{m}_{\mathcal{R}}^{0}=\mathcal{R} \boldsymbol{m}^{0}$. Then

$$
\left|\boldsymbol{m}^{0}-\boldsymbol{Q}\right|^{2}=\left|\mathcal{R}\left(\boldsymbol{m}^{0}-\boldsymbol{Q}\right)\right|^{2}=\left|\boldsymbol{m}_{\mathcal{R}}^{0}-(0,0,-1)\right|^{2}=2\left(1+m_{3, \mathcal{R}}^{0}\right) .
$$

Hence,

$$
\inf _{x \in \mathbb{R}^{N}} m_{3, \mathcal{R}}^{0} \geq-1+\delta
$$

and

$$
\left[\boldsymbol{m}_{\mathcal{R}}^{0}\right]_{B M O}=\left[\boldsymbol{m}^{0}\right]_{B M O} \leq \varepsilon_{0} .
$$

Therefore, Theorem 2.9 provides the existence of a unique smooth solution $\boldsymbol{m}_{\mathcal{R}} \in X\left(\mathbb{R}^{N} \times \mathbb{R}^{+} ; \mathbb{S}^{2}\right)$ of $\left(\mathrm{LLG}_{\alpha}\right)$ satisfying (2.44). Using the invariance of $\left(\mathrm{LLG}_{\alpha}\right)$ and setting $\boldsymbol{m}=\mathcal{R}^{-1} \boldsymbol{m}_{\mathcal{R}}$ we obtain the existence of the desired solution. To establish the uniqueness, it suffices to observe that if $\boldsymbol{n}$ is another smooth solution of $\left(\mathrm{LLG}_{\alpha}\right)$ satisfying $(2.47)$, then $\boldsymbol{n}_{\mathcal{R}}:=\mathcal{R} \boldsymbol{n}$ is a solution of $\left(\mathrm{LLG}_{\alpha}\right)$ with initial condition $\boldsymbol{m}_{\mathcal{R}}^{0}$ and it fulfills (2.44). Therefore, from the uniqueness of solution in Theorem 2.9, it follows that $\boldsymbol{m}_{\mathcal{R}}=\boldsymbol{n}_{\mathcal{R}}$ and then $\boldsymbol{m}=\boldsymbol{n}$.

Proof of Theorem 1.1. In Theorem 2.9 and Corollary 2.11, the constants are given by $C=C_{1} C_{2}^{2}$ and $K=C_{2}$. As discussed in Remark 2.10, the value

$$
\rho^{*}=\frac{\delta^{4}}{32 C_{1} C_{2}^{2}}
$$


maximizes the range for $\varepsilon_{0}$ in (2.27) and this inequality is satisfied for any $\varepsilon_{0}>0$ such that

$$
\varepsilon_{0} \leq \frac{\delta^{6}}{256 C_{1} C_{2}^{2}}
$$

Taking

$$
M_{1}=\frac{1}{256 C_{1} C_{2}^{2}}, \quad M_{2}=C_{2} \quad \text { and } \quad M_{3}=\frac{1}{32 C_{1} C_{2}^{2}},
$$

so that $\rho^{*}=M_{3} \delta^{4}$, the conclusion follows from Theorem 2.9 and Corollary 2.11.

Remark 2.14. We finally remark that is possible to state local (in time) versions of Theorems 2.1 and 2.9 as it was done in $[23,22,35]$. In our context, the local well-posedness would concern solutions with initial condition $\boldsymbol{m}^{0} \in \overline{V M O}\left(\mathbb{R}^{N}\right)$, i.e. such that

$$
\lim _{r \rightarrow 0^{+}} \sup _{x \in \mathbb{R}^{N}} f_{B_{r}(x)}\left|\boldsymbol{m}^{0}(y)-\boldsymbol{m}_{x, r}^{0}\right| d y=0 .
$$

Moreover, some uniqueness results have been established for solutions with this kind of initial data by Miura [32] for the Navier-Stokes equation, and adapted by Lin [29] to (HFHM). It is also possible to do this for $\left(\mathrm{LLG}_{\alpha}\right)$, for $\alpha>0$. We do not pursuit here these types of results because they do not apply to the self-similar solutions $\boldsymbol{m}_{c, \alpha}$. This is due to the facts that the function $\boldsymbol{m}_{\boldsymbol{A}^{ \pm}}^{0}$ does not belong to $\overline{V M O}(\mathbb{R})$ and that

$$
\lim _{T \rightarrow 0^{+}} \sup _{0<t<T} \sqrt{t}\left\|\partial_{x} \boldsymbol{m}_{c, \alpha}\right\|_{L^{\infty}} \neq 0 .
$$

\section{Applications}

\subsection{Existence of self-similar solutions in $\mathbb{R}^{N}$}

The LLG equation is invariant under the scaling $(x, t) \rightarrow\left(\lambda x, \lambda^{2} t\right)$, for $\lambda>0$, that is if $\boldsymbol{m}$ satisfies $\left(L L G_{\alpha}\right)$, then so does the function

$$
\boldsymbol{m}_{\lambda}(x, t)=\boldsymbol{m}\left(\lambda x, \lambda^{2} t\right), \quad \lambda>0 .
$$

Therefore is natural to study the existence of self-similar solutions (of expander type), i.e. a solution $\boldsymbol{m}$ satisfying

$$
\boldsymbol{m}(x, t)=\boldsymbol{m}\left(\lambda x, \lambda^{2} t\right), \quad \forall \lambda>0,
$$

or, equivalently,

$$
\boldsymbol{m}(x, t)=\boldsymbol{f}\left(\frac{x}{\sqrt{t}}\right)
$$

for some $\boldsymbol{f}: \mathbb{R}^{N} \longrightarrow \mathbb{S}^{2}$ profile of $\boldsymbol{m}$. In particular we have the relation $\boldsymbol{f}(y)=\boldsymbol{m}(y, 1)$, for all $y \in \mathbb{R}^{N}$. From (3.1) we see that, at least formally, a necessary condition for the existence of a self-similar solution is that initial condition $\boldsymbol{m}^{0}$ be homogeneous of degree 0, i.e.

$$
\boldsymbol{m}^{0}(\lambda x)=\boldsymbol{m}^{0}(x), \quad \forall \lambda>0 .
$$

Since the norm in $X\left(\mathbb{R}^{N} \times \mathbb{R}^{+} ; \mathbb{R}^{3}\right)$ is invariant under this scaling, i.e.

$$
\left\|\boldsymbol{m}_{\lambda}\right\|_{X}=\|\boldsymbol{m}\|_{X}, \quad \forall \lambda>0,
$$

where $\boldsymbol{m}_{\lambda}$ is defined by (3.1), Theorem 2.9 yields the following result concerning the existence of self-similar solutions. 
Corollary 3.1. With the same notations and hypotheses as in Theorem 2.9, assume also that $\boldsymbol{m}^{0}$ is homogeneous of degree zero. Then the solution $\boldsymbol{m}$ of $\left(\mathrm{LLG}_{\alpha}\right)$ provided by Theorem 2.9 is self-similar. In particular there exists a smooth profile $f: \mathbb{R}^{N} \rightarrow \mathbb{S}^{2}$ such that

$$
\boldsymbol{m}(x, t)=\boldsymbol{f}\left(\frac{x}{\sqrt{t}}\right)
$$

for all $x \in \mathbb{R}^{N}$ and $t>0$, and $\boldsymbol{f}$ satisfies the equation

$$
-\frac{1}{2} y \cdot \nabla \boldsymbol{f}(y)=\beta \boldsymbol{f}(y) \times \Delta \boldsymbol{f}(y)-\alpha \boldsymbol{f}(y) \times(\boldsymbol{f}(y) \times \Delta \boldsymbol{f}(y)),
$$

for all $y \in \mathbb{R}^{N}$. Here $y \cdot \nabla \boldsymbol{f}(y)=\left(y \cdot \nabla f_{1}(y), \ldots, y \cdot \nabla f_{N}(y)\right)$.

Remark 3.2. Analogously, Theorem 2.1 leads to the existence of self-similar solutions for (DNLS), provided that $u^{0}$ is a homogeneous function of degree zero.

For instance, in dimensions $N \geq 2$, Corollary 3.1 applies to the initial condition

$$
\boldsymbol{m}^{0}(x)=\boldsymbol{H}\left(\frac{x}{|x|}\right)
$$

with $\boldsymbol{H}$ a Lipschitz map from $\mathbb{S}^{N-1}$ to $\mathbb{S}^{2} \cap\left\{\left(x_{1}, x_{2}, x_{3}\right): x_{3} \geq-1 / 2\right\}$, provided that the Lipschitz constant is small enough. Indeed, using (2.58), we have

$$
\left[\boldsymbol{m}^{0}\right]_{B M O} \leq 4\|\boldsymbol{H}\|_{\text {Lip }},
$$

so that taking

$$
\delta=1 / 2, \quad \rho=\frac{\delta^{4}}{32 K^{4} C}, \quad \varepsilon_{0}=\frac{\delta^{6}}{256 K^{4} C} \quad \text { and } \quad\|\boldsymbol{H}\|_{\text {Lip }} \leq \varepsilon_{0},
$$

the condition (2.42) is satisfied and we can invoke Corollary 3.1.

Other authors have considered self-similar solutions for the harmonic map flow (i.e. $\left(\mathrm{LLG}_{\alpha}\right)$ with $\alpha=1$ ) in different settings. Actually, equation (HFHM) can be generalized for maps $\boldsymbol{m}: \mathcal{M} \times \mathbb{R}^{+} \rightarrow \mathcal{N}$, with $\mathcal{M}$ and $\mathcal{N}$ Riemannian manifolds. Biernat and Bizoń [8] established results when $\mathcal{M}=\mathcal{N}=\mathbb{S}^{d}$ and $3 \leq d \leq 6$. Also, Germain and Rupflin [15] have investigated the case $\mathcal{M}=\mathbb{R}^{d}$ and $\mathcal{N}=\mathbb{S}^{d}$, in $d \geq 3$. In both works the analysis is done only for equivariant solutions and does not cover the case $\mathcal{M}=\mathbb{R}^{N}$ and $\mathcal{N}=\mathbb{S}^{2}$.

\subsection{The Cauchy problem for the one-dimensional LLG equation with a jump initial data}

This section is devoted to prove Theorems 1.2 and 1.3 in the introduction. These two results concern the question of well-posedness/ill-posedness of the Cauchy problem for the one-dimensional LLG equation associated with a step function initial condition of the form

$$
m_{A^{ \pm}}^{0}:=A^{+} \chi_{\mathbb{R}^{+}}+A^{-} \chi_{\mathbb{R}^{-}},
$$

where $\boldsymbol{A}^{+}$and $\boldsymbol{A}^{-}$are two given unitary vectors in $\mathbb{S}^{2}$. 


\subsubsection{Existence, uniqueness and stability. Proof of Theorem 1.2}

As mentioned in the introduction, in [17] we proved the existence of the uniparametric smooth family of self-similar solutions $\left\{\boldsymbol{m}_{c, \alpha}\right\}_{c>0}$ of $\left(\mathrm{LLG}_{\alpha}\right)$ for all $\alpha \in[0,1]$ with initial condition of the type (3.2) given by

$$
\boldsymbol{m}_{c, \alpha}^{0}:=\boldsymbol{A}_{c, \alpha}^{+} \chi_{\mathbb{R}^{+}}+\boldsymbol{A}_{c, \alpha}^{-} \chi_{\mathbb{R}^{-}},
$$

where $\boldsymbol{A}_{c, \alpha}^{ \pm} \in \mathbb{S}^{2}$ are given by Theorem A.5. For the convenience of the reader, we collect some of the results proved in [17] in the Appendix. The results in this section rely on a further understanding of the properties of the self-similar solutions $\boldsymbol{m}_{c, \alpha}$.

In Proposition 3.4 we show that

$$
\boldsymbol{m}_{c, \alpha}=\left(m_{1, c, \alpha}, m_{2, c, \alpha}, m_{3, c, \alpha}\right) \in X\left(\mathbb{R} \times \mathbb{R}^{+} ; \mathbb{S}^{2}\right),
$$

that $m_{3, c, \alpha}$ is far from the South Pole and that $\left[\boldsymbol{m}_{c, \alpha}\right]_{X}$ is small, if $c$ is small enough. This will yield that $\boldsymbol{m}_{c, \alpha}$ corresponds (up to a rotation) to the solution given by Corollary 3.1. More precisely, using the invariance under rotations of $\left(\mathrm{LLG}_{\alpha}\right)$, we can prove that, if the angle between $\boldsymbol{A}^{+}$and $\boldsymbol{A}^{-}$is small enough, then the solution given by Corollary 3.1 with initial condition $\boldsymbol{m}_{\boldsymbol{A}^{ \pm}}^{0}$ coincides (modulo a rotation) with $\boldsymbol{m}_{c, \alpha}$, for some $c$. We have the following:

Theorem 3.3. Let $\alpha \in(0,1]$. There exist $L_{1}, L_{2}>0, \delta^{*} \in(-1,0)$ and $\vartheta^{*}>0$ such that the following holds. Let $\boldsymbol{A}^{+}, \boldsymbol{A}^{-} \in \mathbb{S}^{2}$ and let $\vartheta$ be the angle between them. If

$$
0<\vartheta \leq \vartheta^{*}
$$

then there exists a solution $\boldsymbol{m}$ of $\left(\mathrm{LLG}_{\alpha}\right)$ with initial condition $\boldsymbol{m}_{\boldsymbol{A} \pm}^{0}$. Moreover, there exists $0<c<\frac{\sqrt{\alpha}}{2 \sqrt{\pi}}$, such that $\boldsymbol{m}$ coincides up to a rotation with the self-similar solution $\boldsymbol{m}_{c, \alpha}$, i.e. there exists $\mathcal{R} \in S O(3)$, depending only on $\boldsymbol{A}^{+}, \boldsymbol{A}^{-}, \alpha$ and $c$, such that

$$
\boldsymbol{m}=\mathcal{R} \boldsymbol{m}_{c, \alpha},
$$

and $\boldsymbol{m}$ is the unique solution satisfying

$$
\inf _{\substack{x \in \mathbb{R} \\ t>0}} m_{3}(x, t) \geq \delta^{*} \quad \text { and } \quad[\boldsymbol{m}]_{X} \leq L_{1}+L_{2} c
$$

In order to prove Theorem 3.3, we need some preliminary estimates for $\boldsymbol{m}_{c, \alpha}$ in terms of $c$ and $\alpha$. To obtain them, we use some properties of the profile profile $\boldsymbol{f}_{c, \alpha}=\left(f_{1, c, \alpha}, f_{2, c, \alpha}, f_{3, c, \alpha}\right)$ constructed in [17] using the Serret-Frenet equations with initial conditions

$$
f_{1, c, \alpha}(0)=1, \quad f_{2, c, \alpha}(0)=f_{3, c, \alpha}(0)=0 .
$$

Also,

$$
\left|f_{j, c, \alpha}^{\prime}(s)\right| \leq c e^{-\alpha s^{2} / 4}, \text { for all } s \in \mathbb{R}
$$

for $j \in\{1,2,3\}$ and

$$
\boldsymbol{m}_{c, \alpha}(x, t)=\boldsymbol{f}_{c, \alpha}\left(\frac{x}{\sqrt{t}}\right), \quad \text { for all }(x, t) \in \mathbb{R} \times \mathbb{R}^{+} .
$$

Hence, for any $x \in \mathbb{R}$,

$$
\left|f_{3, c_{\alpha}}(x)\right|=\left|f_{3, c_{\alpha}}(x)-f_{3, c_{\alpha}}(0)\right| \leq \int_{0}^{|x|} c e^{-\alpha \sigma^{2} / 4} d \sigma \leq c \frac{\sqrt{\pi}}{\sqrt{\alpha}} .
$$


Since the same estimate holds for $f_{2, c, \alpha}$, we conclude that

$$
\left|m_{2, c, \alpha}(x, t)\right| \leq c \frac{\sqrt{\pi}}{\sqrt{\alpha}}, \quad \text { and } \quad\left|m_{3, c, \alpha}(x, t)\right| \leq c \frac{\sqrt{\pi}}{\sqrt{\alpha}} \quad \text { for all }(x, t) \in \mathbb{R} \times \mathbb{R}^{+} .
$$

Moreover, since

$$
\boldsymbol{A}_{c, \alpha}^{ \pm}=\lim _{x \rightarrow \pm \infty} \boldsymbol{f}_{c, \alpha}(x)
$$

we also get

$$
\left|A_{j, c, \alpha}^{ \pm}\right| \leq c \frac{\sqrt{\pi}}{\sqrt{\alpha}}, \quad \text { for } j \in\{2,3\} .
$$

We now provide some further properties of the self-similar solutions.

Proposition 3.4. For $\alpha \in(0,1]$ and $c>0$, we have

$$
\begin{gathered}
\left\|m_{2, c, \alpha}^{0}\right\|_{L^{\infty}} \leq c \frac{\sqrt{\pi}}{\sqrt{\alpha}}, \quad\left\|m_{3, c, \alpha}^{0}\right\|_{L^{\infty}} \leq c \frac{\sqrt{\pi}}{\sqrt{\alpha}}, \quad \sup _{t>0}\left\|m_{3, c, \alpha}\right\|_{L^{\infty}} \leq c \frac{\sqrt{\pi}}{\sqrt{\alpha}}, \\
{\left[\boldsymbol{m}_{c, \alpha}^{0}\right]_{B M O} \leq 2 c \frac{\sqrt{2 \pi}}{\sqrt{\alpha}},} \\
\sqrt{t}\left\|\partial_{x} \boldsymbol{m}_{c, \alpha}\right\|_{\infty}=c, \quad \text { for all } t>0, \\
\sup _{\substack{x \in \mathbb{R} \\
r>0}} \frac{1}{r} \int_{Q_{r}(x)}\left|\partial_{y} \boldsymbol{m}_{c, \alpha}(y, t)\right|^{2} d t d y \leq \frac{2 \sqrt{2 \pi} c^{2}}{\sqrt{\alpha}} .
\end{gathered}
$$

In particular, $\boldsymbol{m}_{c, \alpha} \in X\left(\mathbb{R} \times \mathbb{R}^{+} ; \mathbb{S}^{2}\right)$ and

$$
\left[\boldsymbol{m}_{c, \alpha}\right]_{X} \leq \frac{4 c}{\alpha^{\frac{1}{4}}} .
$$

Proof of Proposition 3.4. The estimates in (3.10) follow from (3.8) and (3.9). To prove (3.11), we use (2.58), (3.3), (3.10) and the fact that

$$
\boldsymbol{A}_{c, \alpha}^{-}=\left(A_{1, c, \alpha}^{+},-A_{2, c, \alpha}^{+},-A_{3, c, \alpha}^{+}\right),
$$

(see Theorem A.5) to get

$$
\begin{aligned}
{\left[\boldsymbol{m}_{c, \alpha}^{0}\right]_{B M O} } & \leq \sup _{x \in \mathbb{R}^{N}} f_{B_{r}(x)} f_{B_{r}(x)}\left|\boldsymbol{m}_{c, \alpha}^{0}(y)-\boldsymbol{m}_{c, \alpha}^{0}(z)\right| d y d z \\
& \leq 2 \sqrt{\left(A_{2, c, \alpha}^{+}\right)^{2}+\left(A_{3, c, \alpha}^{+}\right)^{2}} \sup _{x \in \mathbb{R}^{N}} f_{B_{r}(x)} f_{B_{r}(x)} d y d z \\
& \leq \frac{2 c \sqrt{2 \pi}}{\sqrt{\alpha}}
\end{aligned}
$$

From (A.12) we obtain the equality in (3.12) and also

$$
I_{r, x}:=\frac{1}{r} \int_{Q_{r}(x)}\left|\partial_{y} \boldsymbol{m}_{c, \alpha}(y, t)\right|^{2} d t d y=\frac{c^{2}}{r} \int_{x-r}^{x+r} \int_{0}^{r^{2}} \frac{e^{\frac{-\alpha y^{2}}{2 t}}}{t} d t d y .
$$

Performing the change of variables $z=\left(\alpha y^{2}\right) /(2 t)$, we see that

$$
\int_{0}^{r^{2}} \frac{e^{\frac{-\alpha y^{2}}{2 t}}}{t} d t=E_{1}\left(\frac{\alpha y^{2}}{2 r^{2}}\right)
$$


where $E_{1}$ is the exponential integral function

$$
E_{1}(y)=\int_{y}^{\infty} \frac{e^{-z}}{z} d z
$$

This function satisfies that $\lim _{y \rightarrow 0^{+}} E_{1}(y)=\infty$ and $\lim _{y \rightarrow \infty} E_{1}(y)=0$ (see e.g. [1, Chapter 5]). Moreover, taking $\epsilon>0$ and integrating by parts,

$$
\int_{\epsilon}^{\infty} E_{1}\left(y^{2}\right) d y=\left.y E_{1}\left(y^{2}\right)\right|_{\epsilon} ^{\infty}+2 \int_{\epsilon}^{\infty} e^{-y^{2}} d y
$$

so L'Hôpital's rule shows that the first term in the r.h.s. of (3.18) vanishes as $\epsilon \rightarrow 0^{+}$. Therefore, the Lebesgue's monotone convergence theorem allows to conclude that $E_{1}\left(y^{2}\right) \in L^{1}\left(\mathbb{R}^{+}\right)$and

$$
\int_{0}^{\infty} E_{1}\left(y^{2}\right)=\sqrt{\pi}
$$

By using (3.16), (3.17), (3.19), and making the change of variables $z=\sqrt{\alpha} y /(r \sqrt{2})$, we obtain

$$
I_{r, x}=\frac{c^{2}}{r} \int_{x-r}^{x+r} E_{1}\left(\frac{\alpha y^{2}}{2 r^{2}}\right) d y=\frac{\sqrt{2} c^{2}}{\sqrt{\alpha}} \int_{\frac{\sqrt{\alpha}}{\sqrt{2}}\left(\frac{x}{r}-1\right)}^{\frac{\sqrt{\alpha}}{\sqrt{2}}\left(\frac{x}{r}+1\right)} E_{1}\left(z^{2}\right) d z \leq \frac{\sqrt{2} c^{2}}{\sqrt{\alpha}} \cdot 2 \sqrt{\pi},
$$

which leads to (3.13). Finally, the bound in (3.14) easily follows from those in (3.12) and (3.13) and the elementary inequality

$$
\left(1+\left(\frac{2 \sqrt{2 \pi}}{\sqrt{\alpha}}\right)^{1 / 2}\right) \leq \frac{1}{\alpha^{\frac{1}{4}}}\left(1+(2 \sqrt{2 \pi})^{1 / 2}\right) \leq \frac{4}{\alpha^{\frac{1}{4}}}, \quad \alpha \in(0,1] .
$$

Proof of Theorem 3.3. First, we consider the case when $\boldsymbol{A}^{+}=\boldsymbol{A}_{c, \alpha}^{+}$and $\boldsymbol{A}^{-}=\boldsymbol{A}_{c, \alpha}^{-}$(i.e. when $\boldsymbol{m}_{\boldsymbol{A}^{ \pm}}^{0}=\boldsymbol{m}_{c, \alpha}^{0}$ ) for some $c>0$. We will continue to show that the solution provided by Theorem 2.9 is exactly $\boldsymbol{m}_{c, \alpha}$, for $c$ small. Indeed, bearing in mind the estimates in Proposition 3.4, we consider

$$
c \leq \frac{\sqrt{\alpha}}{2 \sqrt{\pi}}
$$

so that

$$
\inf _{x \in \mathbb{R}} m_{3, c, \alpha}^{0}(x) \geq-\frac{1}{2}
$$

In view of (3.11), (3.21) and Remark 2.10, we set

$$
\varepsilon_{0}:=4 c \frac{\sqrt{\pi}}{\sqrt{\alpha}}, \quad \delta:=\frac{1}{2}, \quad \rho:=\frac{\delta^{4}}{8 K^{4} C}=\frac{1}{2^{7} K^{4} C},
$$

where $C, K \geq 1$ are the constants given by Theorem 2.9. In this manner, from (3.11), (3.21) and (3.22), we have

$$
\inf _{\mathbb{R}} m_{3}^{0} \geq-1+\delta \quad \text { and } \quad\left[\boldsymbol{m}^{0}\right]_{B M O} \leq \varepsilon_{0},
$$

and the condition (2.42) is fulfilled if

$$
\varepsilon_{0} \leq \frac{\delta^{6}}{256 K^{4} C}
$$

or equivalently, if $c \leq \tilde{c}$, with

$$
\tilde{c}:=\frac{\sqrt{\alpha}}{2^{16} K^{4} C \sqrt{\pi}}
$$


Observe that in particular $\tilde{c}<\frac{\sqrt{\alpha}}{2 \sqrt{\pi}}$.

For fixed $0<c<\tilde{c}$, we can apply Theorem 2.9 to deduce the existence and uniqueness of a solution $\boldsymbol{m}$ of $\left(\mathrm{LLG}_{\alpha}\right)$ satisfying

$$
\inf _{\substack{x \in \mathbb{R} \\ t>0}} m_{3}(x, t) \geq-1+\frac{2}{1+K^{2}(\rho+2)^{2}} \quad \text { and } \quad[\boldsymbol{m}]_{X} \leq 4 K \rho+\frac{2^{9} K c \sqrt{\pi}}{\sqrt{\alpha}} .
$$

Now by Proposition 3.4, for fixed $0<c \leq \tilde{c}$, we have the following estimates for $\boldsymbol{m}_{c, \alpha}$

$$
\left[\boldsymbol{m}_{c, \alpha}\right]_{X} \leq 4 c / \alpha^{\frac{1}{4}} \quad \text { and } \quad \inf _{\substack{x \in \mathbb{R} \\ t>0}} m_{3, c, \alpha}(x, t) \geq-\frac{1}{2},
$$

so in particular $\boldsymbol{m}_{c, \alpha}$ satisfies (3.23). Thus the uniqueness of solution implies that $\boldsymbol{m}=\boldsymbol{m}_{c, \alpha}$, provided that $c \leq \tilde{c}$. Defining the constants $L_{1}, L_{2}$ and $\delta^{*}$ by

$$
L_{1}=4 K \rho, \quad L_{2}=\frac{2^{9} K \sqrt{\pi}}{\sqrt{\alpha}} \quad \text { and } \quad \delta^{*}=-1+\frac{2}{1+K^{2}(\rho+2)^{2}},
$$

the theorem is proved in the case $\boldsymbol{A}^{ \pm}=\boldsymbol{A}_{c, \alpha}^{ \pm}$.

For the general case, we would like to understand which angles can be reached by varying the parameter $c$ in the range $(0, \tilde{c}]$. To this end, for fixed $0<c \leq \tilde{c}$, let $\vartheta_{c, \alpha}$ be the angle between $\boldsymbol{A}_{c, \alpha}^{+}$and $\boldsymbol{A}_{c, \alpha}^{-}$. From Lemma A.6,

$$
\vartheta_{c, \alpha} \geq \arccos \left(1-c^{2} \pi+32 \frac{c^{3} \sqrt{\pi}}{\alpha^{2}}\right), \quad \text { for all } c \in\left(0, \frac{\alpha^{2} \sqrt{\pi}}{32}\right] .
$$

Now, it is easy to see that the function $F(c)=\arccos \left(1-c^{2} \pi+32 \frac{c^{3} \sqrt{\pi}}{\alpha^{2}}\right)$ is strictly increasing on the interval $\left[0, \alpha^{2} \frac{\sqrt{\pi}}{48}\right]$ so that

$$
F(c)>F(0)=0, \quad \text { for all } c \in\left(0, \frac{\alpha^{2} \sqrt{\pi}}{48}\right] .
$$

Let $c^{*}=\min \left(\tilde{c}, \frac{\alpha^{2} \sqrt{\pi}}{48}\right)$ and consider the map $T_{\alpha}: c \longrightarrow \vartheta_{c, \alpha}$ on $\left[0, c^{*}\right]$. By Lemma A.6, $T_{\alpha}$ is continuous on $\left[0, c^{*}\right], T_{\alpha}(0)=\lim _{c \rightarrow 0^{+}} T_{\alpha}(c)=0$ and, bearing in mind $(3.25), T\left(c^{*}\right)=\vartheta_{c^{*}, \alpha}>0$. Thus, from the intermediate value theorem we infer that for any $\vartheta \in\left(0, \vartheta_{c^{*}, \alpha}\right)$, there exists $c \in\left(0, c^{*}\right)$ such that

$$
\vartheta=T_{\alpha}(c)=\vartheta_{c, \alpha}
$$

We can now complete the proof for any $\boldsymbol{A}^{+}, \boldsymbol{A}^{-} \in \mathbb{S}^{2}$. Let $\vartheta$ be the angle between $\boldsymbol{A}^{+}$and $\boldsymbol{A}^{-}$. From the previous lines, we know that there exists $\vartheta^{*}:=\vartheta_{c^{*}, \alpha}$ such that if $\vartheta \in\left(0, \vartheta^{*}\right)$, there exists $c \in\left(0, c^{*}\right)$ such that $\vartheta=\vartheta_{c, \alpha}$. For this value of $c$, consider the initial value problem associated with $\boldsymbol{m}_{c, \alpha}^{0}$ and the constants defined in (3.24). We have already seen the existence of a unique solution $\boldsymbol{m}_{c, \alpha}$ of the LLG equation associated with this initial condition satisfying (3.6). Let $\mathcal{R} \in S O(3)$ be the rotation on $\mathbb{R}^{3}$ such that $\boldsymbol{A}^{+}=\mathcal{R} \boldsymbol{A}_{c, \alpha}^{+}$and $\boldsymbol{A}^{-}=\mathcal{R} \boldsymbol{A}_{c, \alpha}^{-}$. Then $\boldsymbol{m}:=\mathcal{R} \boldsymbol{m}_{c, \alpha}$ solves $\left(\mathrm{LLG}_{\alpha}\right)$ with initial condition $\boldsymbol{m}_{\boldsymbol{A}^{ \pm}}^{0}$. Finally, recalling the above definition of $L_{1}, L_{2}$ and $\delta^{*}$, using the invariance of the norms under rotations and the fact that $\boldsymbol{m}_{c, \alpha}$ is the unique solution satisfying (3.23), it follows that $\boldsymbol{m}$ is the unique solution satisfying the conditions in the statement of the theorem.

We are now in position to give the proof of Theorem 1.2, the second of our main results in this paper. In fact, we will see that Theorem 1.2 easily follows from Theorem 3.3 and the well-posedness for the LLG equation stated in Theorem 2.9. 
Proof of Theorem 1.2. Let $\vartheta^{*}, \delta^{*}, L_{1}$ and $L_{2}$ be the constants defined in the proof of Theorem 3.3. Given $\boldsymbol{A}^{+}$and $\boldsymbol{A}^{-}$such that $0<\vartheta<\vartheta^{*}$, Theorem 3.3 asserts the existence of

$$
0<c<\frac{\sqrt{\alpha}}{2 \sqrt{\pi}}
$$

and $\mathcal{R} \in S O(3)$ such that $\mathcal{R} \boldsymbol{m}_{c, \alpha}$ is the unique solution of $\left(\mathrm{LLG}_{\alpha}\right)$ with initial condition $\boldsymbol{m}_{\boldsymbol{A}^{ \pm}}^{0}$ satisfying (3.6), and in particular $\boldsymbol{m}_{\boldsymbol{A}^{ \pm}}^{0}=\mathcal{R} \boldsymbol{m}_{c, \alpha}^{0}$. By hypothesis $\boldsymbol{m}^{0}$ satisfies

$$
\left\|\boldsymbol{m}^{0}-\boldsymbol{m}_{\boldsymbol{A}^{ \pm}}^{0}\right\|_{L^{\infty}} \leq \frac{c \sqrt{\pi}}{2 \sqrt{\alpha}}
$$

Hence, defining $\boldsymbol{m}_{\mathcal{R}}^{0}=\mathcal{R}^{-1} \boldsymbol{m}^{0}$, recalling that $[f]_{B M O} \leq 2\|f\|_{L^{\infty}}$ and using the invariance of the norms under rotations, we deduce from (3.27) that

$$
\left\|\boldsymbol{m}_{\mathcal{R}}^{0}\right\|_{L^{\infty}} \leq\left\|\boldsymbol{m}_{c, \alpha}^{0}\right\|_{L^{\infty}}+\frac{c \sqrt{\pi}}{2 \sqrt{\alpha}} \quad \text { and } \quad\left[\boldsymbol{m}_{\mathcal{R}}^{0}\right]_{B M O} \leq\left[\boldsymbol{m}_{c, \alpha}^{0}\right]_{B M O}+\frac{c \sqrt{\pi}}{\sqrt{\alpha}} .
$$

Then, by Proposition 3.4,

$$
\left\|\boldsymbol{m}_{3, \mathcal{R}}^{0}\right\|_{L^{\infty}} \leq \frac{2 c \sqrt{\pi}}{\sqrt{\alpha}} \quad \text { and } \quad\left[\boldsymbol{m}_{\mathcal{R}}^{0}\right]_{B M O} \leq \frac{4 c \sqrt{\pi}}{\sqrt{\alpha}} .
$$

From (3.26) and (3.28), it follows that

$$
\boldsymbol{m}_{3, \mathcal{R}}^{0}(x) \geq-1 / 2, \quad \text { for all } x \in \mathbb{R} .
$$

Therefore, as in the proof of Theorem 3.3, we can apply Theorem 2.9 with the values of $\varepsilon_{0}, \delta$ and $\rho$ given in (3.22) to deduce the existence of a unique (smooth) solution $\boldsymbol{m}_{\mathcal{R}}$ of $\left(L L G_{\alpha}\right)$ with initial condition $\boldsymbol{m}_{\mathcal{R}}^{0}$ satisfying

$$
\inf _{\substack{x \in \mathbb{R} \\ t>0}} \boldsymbol{m}_{3, \mathcal{R}}(x, t) \geq-1+\frac{2}{1+K^{2}(\rho+2)^{2}}=\delta^{*} \quad \text { and } \quad\left[\boldsymbol{m}_{\mathcal{R}}\right]_{X} \leq 4 K \rho+\frac{2^{9} K c \sqrt{\pi}}{\sqrt{\alpha}}=L_{1}+L_{2} c .
$$

Since we have taken the values for $\varepsilon_{0}, \delta$ and $\rho$ as in the proof Theorem 3.3, Theorem 2.9 also implies that

$$
\left\|\boldsymbol{m}_{\mathcal{R}}-\boldsymbol{m}_{c, \alpha}\right\|_{X} \leq 480 K\left\|\boldsymbol{m}_{\mathcal{R}}^{0}-\boldsymbol{m}_{c, \alpha}^{0}\right\|_{L^{\infty}} .
$$

The conclusion of the theorem follows defining $\boldsymbol{m}=\mathcal{R} \boldsymbol{m}_{\mathcal{R}}$ and $L_{3}=480 \mathrm{~K}$, and using once again the invariance of the norm under rotations.

\subsubsection{Multiplicity of solutions. Proof of Theorem 1.3}

As proved in [17], when $\alpha=1$, the self-similar solutions are explicitly given by

$$
\boldsymbol{m}_{c, 1}(x, t)=(\cos (c \operatorname{Erf}(x / \sqrt{t})), \sin (c \operatorname{Erf}(x / \sqrt{t})), 0), \quad \text { for all }(x, t) \in \mathbb{R} \times \mathbb{R}^{+},
$$

for every $c>0$, where $\operatorname{Erf}(\cdot)$ is the non-normalized error function

$$
\operatorname{Erf}(s)=\int_{0}^{s} e^{-\sigma^{2} / 4} d \sigma
$$

In particular,

$$
\overrightarrow{\boldsymbol{A}}_{c, 1}^{ \pm}=(\cos (c \sqrt{\pi}), \pm \sin (c \sqrt{\pi}), 0)
$$




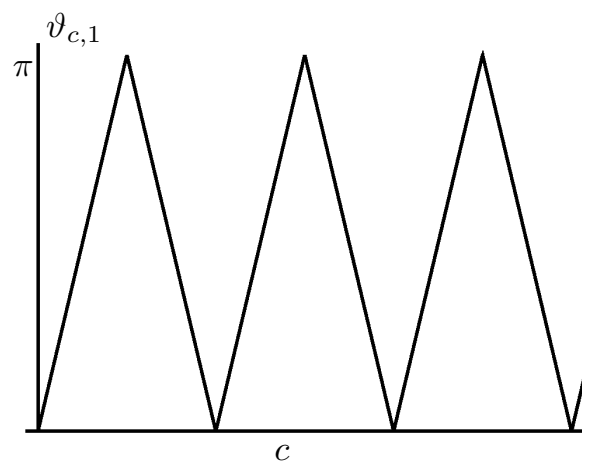

Figure 2: The angle $\vartheta_{c, \alpha}$ as a function of $c$ for $\alpha=1$.

and the angle between $\boldsymbol{A}_{c, 1}^{+}$and $\boldsymbol{A}_{c, 1}^{-}$is given by

$$
\vartheta_{c, 1}=\arccos (\cos (2 c \sqrt{\pi})) \text {. }
$$

Formula (3.30) and Figure 2 show that there are infinite values of $c$ that allow to reach any angle in $[0, \pi]$. Therefore, using the invariance of $\left(\mathrm{LLG}_{\alpha}\right)$ under rotations, in the case when $\alpha=1$, one can easily prove the existence of multiple solutions associated with a given initial data of the form $\boldsymbol{m}_{\boldsymbol{A}^{ \pm}}^{0}$ for any given vectors $\boldsymbol{A}^{ \pm} \in \mathbb{S}^{2}$ (see argument in the proof included below). In the case that $\alpha$ is close enough to 1 , we can use a continuity argument to prove that we still have multiple solutions. More precisely, Theorem 1.3 asserts that for any given initial data of the form $\boldsymbol{m}_{\boldsymbol{A}^{ \pm}}^{0}$ with angle between $\boldsymbol{A}^{+}$and $\boldsymbol{A}^{-}$in the interval $(0, \pi)$, if $\alpha$ is sufficiently close to one, then there exist at least $k$-distinct solutions of $\left(\mathrm{LLG}_{\alpha}\right)$ associated with the same initial condition, for any given $k \in \mathbb{N}$.

The rest of this section is devoted to the proof of Theorem 1.3.

Proof of Theorem 1.3. Let $k \in \mathbb{N}, \boldsymbol{A}^{ \pm} \in \mathbb{S}^{2}$ and $\vartheta \in(0, \pi)$ be the angle between $\boldsymbol{A}^{+}$and $\boldsymbol{A}^{-}$. Using the invariance of $\left(\mathrm{LLG}_{\alpha}\right)$ under rotations, it suffices to prove the existence of $\alpha_{k} \in(0,1)$ such that for every $\alpha \in\left[\alpha_{k}, 1\right]$ there exist $0<c_{1}<\cdots<c_{k}$ such that the angle $\vartheta_{c_{j}, \alpha}$ between $\boldsymbol{A}_{c_{j}, \alpha}^{+}$and $\boldsymbol{A}_{c_{j}, \alpha}^{-}$, satisfies

$$
\vartheta_{c_{j}, \alpha}=\vartheta, \quad \text { for all } j \in\{1, \ldots, k\} .
$$

In what follows, and since we want to show the existence of at least $k$-distinct solutions, we will assume without loss of generality that $k$ is large enough.

First observe that, since $\boldsymbol{A}_{c, \alpha}^{-}=\left(A_{1, c, \alpha}^{+},-A_{2, c, \alpha}^{+},-A_{3, c, \alpha}^{+}\right)$, we have the explicit formula

$$
\cos \left(\vartheta_{c, \alpha}\right)=2\left(A_{1, c, \alpha}^{+}\right)^{2}-1 \text {, }
$$

and using Lemma A.8 in the Appendix, we get

$$
\left|\cos \left(\vartheta_{c, \alpha}\right)-\cos \left(\vartheta_{c, 1}\right)\right|=\left|2\left(\left(A_{1, c, \alpha}^{+}\right)^{2}-\left(A_{1, c, 1}^{+}\right)^{2}\right)\right| \leq 4\left|A_{1, c, \alpha}^{+}-A_{1, c, 1}^{+}\right| \leq 4 h(c) \sqrt{1-\alpha},
$$

for all $\alpha \in[1 / 2,1]$, with $h: \mathbb{R}^{+} \longrightarrow \mathbb{R}^{+}$an increasing function satisfying $\lim _{s \rightarrow \infty} h(s)=\infty$.

For $j \in \mathbb{N}$, we set $a_{j}=(2 j+1) \sqrt{\pi} / 2$ and $b_{j}=(2 j+2) \sqrt{\pi} / 2$, so that (3.30) and (3.32) yield

$$
\cos \left(\vartheta_{a_{j}, \alpha}\right) \leq-1+4 h\left(a_{j}\right) \sqrt{1-\alpha} \quad \text { and } \quad \cos \left(\vartheta_{b_{j}, \alpha}\right) \geq 1-4 h\left(b_{j}\right) \sqrt{1-\alpha}, \quad \forall \alpha \in[1 / 2,1] .
$$


Define $l=\cos (\vartheta)$ and

$$
\alpha_{k}=\max \left(1-\left(\frac{1-l}{8 h\left(b_{k}\right)}\right)^{2}, 1-\left(\frac{1+l}{8 h\left(b_{k}\right)}\right)^{2}\right) .
$$

Notice that, since $\vartheta \in(0, \pi)$, we have $-1<l<1$ and thus $\alpha_{k}<1$. Also, since $h$ diverges to $\infty$, we can assume without loss of generality that $\alpha_{k} \in[1 / 2,1)$, and from the definition of $\alpha_{k}$ we have

$$
0<\sqrt{1-\alpha_{k}}<\min \left(\frac{1-l}{8 h\left(b_{k}\right)}, \frac{1+l}{8 h\left(b_{k}\right)}\right) .
$$

Therefore, from (3.33) and $h\left(a_{j}\right)<h\left(b_{j}\right) \leq h\left(b_{k}\right)$ (since $h$ is a strictly increasing function), we get

$$
\cos \left(\vartheta_{a_{j}, \alpha}\right) \leq \frac{-1+l}{2} \quad \text { and } \quad \frac{1+l}{2} \leq \cos \left(\vartheta_{b_{j}, \alpha}\right), \quad \forall j \in\{1, \ldots, k\}, \forall \alpha \in\left[\alpha_{k}, 1\right],
$$

and thus

$$
\cos \left(\vartheta_{a_{j}, \alpha}\right)<l<\cos \left(\vartheta_{b_{j}, \alpha}\right), \quad \forall j \in\{1, \ldots, k\}, \forall \alpha \in\left[\alpha_{k}, 1\right],
$$

since $l \in(-1,1)$.

Let us fix $\alpha \in\left[\alpha_{k}, 1\right]$ and $j \in\{1, \ldots, k\}$. By Lemma A.8, $c \rightarrow \cos \left(\vartheta_{c, \alpha}\right)$ is a continuous function on $c$. Therefore (3.34) and the intermediate value theorem yield the existence of $c_{j} \in$ $\left[a_{j}, b_{j}\right]$ such that

$$
\cos \left(\vartheta_{c_{j}, \alpha}\right)=l=\cos (\vartheta)
$$

or equivalently, such that $\vartheta_{c_{j}, \alpha}=\vartheta$.

Finally, for each $j \in\{1, \ldots, k\}$, let $\mathcal{R}_{j} \in S O(3)$ be such that $\boldsymbol{m}_{A^{ \pm}}^{0}=\mathcal{R}_{j} \boldsymbol{m}_{c_{j}, \alpha}^{0}$, and define $\boldsymbol{m}_{j}$ by $\boldsymbol{m}_{j}=\mathcal{R}_{j} \boldsymbol{m}_{c_{j}, \alpha}$. Then $\boldsymbol{m}_{j}$ solves $\left(\mathrm{LLG}_{\alpha}\right)$ with initial data $\boldsymbol{m}_{\boldsymbol{A}^{ \pm}}^{0}$ and the control of $\partial_{x} \boldsymbol{m}_{j}$ in (1.9) follows from the definition of $\boldsymbol{m}_{j}$ in terms of $\boldsymbol{m}_{c_{j}, \alpha}$ and the analogous property established in Theorem A.5 for the self-similar solution $\boldsymbol{m}_{c_{j}, \alpha}$ (see (A.12)).

In the case when $\alpha=1$ and $\vartheta \in[0, \pi]$, formula (3.30) shows that sequence $\left\{c_{j}\right\}_{j \geq 1}$ in (1.10) satisfies $\vartheta_{c_{j}, 1}=\vartheta$ for all $j \in \mathbb{N}^{*}$. The result follows by considering the sequence of solutions $\left\{\boldsymbol{m}_{j}\right\}_{j \geq 1}$ described above.

Remark 3.5. Notice that the proof given above also shows how close $\alpha_{k}$ needs to be to 1 in terms of the (fixed) angle $\vartheta \in(0, \pi)$, and in particular $\alpha_{k} \rightarrow 1$ as $k \rightarrow \infty$, and $\alpha_{k} \rightarrow 1$ as $\vartheta \rightarrow 0$ (i.e. when $l \rightarrow 1$ ).

Remark 3.6. For $\alpha=1$ and $c>0$, the function

$$
u(x, t)=\mathcal{P}\left(\boldsymbol{m}_{c, 1}\right)=\exp (i c \operatorname{Erf}(x / \sqrt{t}))
$$

is a solution of (DNLS) with initial condition

$$
u^{0}=e^{i c \sqrt{\pi}} \chi_{\mathbb{R}^{+}}+e^{-i c \sqrt{\pi}} \chi_{\mathbb{R}^{-}} .
$$

Therefore there is also a multiplicity phenomenon for the equation (DNLS).

\subsection{A singular solution for a nonlocal Schrödinger equation}

We have used the stereographic projection to establish a well-posedness result for ( $\left.\mathrm{LLG}_{\alpha}\right)$. Melcher [31] showed a global well-posedness result, provided that

$$
\left\|\nabla \boldsymbol{m}^{0}\right\|_{L^{N}} \leq \varepsilon, \quad \boldsymbol{m}^{0}-\boldsymbol{Q} \in H^{1}\left(\mathbb{R}^{N}\right) \cap W^{1, N}\left(\mathbb{R}^{N}\right), \quad \alpha>0, \quad N \geq 3,
$$


for some $\boldsymbol{Q} \in \mathbb{S}^{2}$ and $\varepsilon>0$ small. Later, Lin, Lan and Wang [30] improved this result and proved global well-posedness under the conditions

$$
\left\|\nabla \boldsymbol{m}^{0}\right\|_{M^{2,2}} \leq \varepsilon, \quad \boldsymbol{m}^{0}-\boldsymbol{Q} \in L^{2}\left(\mathbb{R}^{N}\right), \quad \alpha>0, \quad N \geq 2,
$$

for some $\boldsymbol{Q} \in \mathbb{S}^{2}$ and $\varepsilon>0$ small. ${ }^{6}$ In the context of Theorem 1.1 and using the characterization of $B M O^{-1}$ in Theorem A.1, the second condition in (1.3) says that $\left\|\nabla \boldsymbol{m}^{0}\right\|_{B M O^{-1}}$ is small. In view of the embeddings

$$
L^{N}\left(\mathbb{R}^{N}\right) \subset M^{2,2}\left(\mathbb{R}^{N}\right) \subset B M O^{-1}\left(\mathbb{R}^{N}\right),
$$

for $N \geq 2$, we deduce that Theorem 1.1 includes initial conditions with less regularity, as long as their essential range is not $\mathbb{S}^{2}$. The argument in $[30,31]$ is based on the method of moving frames that produces a covariant complex Ginzburg-Landau equation. One of the aims of this subsection is to compare their approach in the context of the self-similar solutions $\boldsymbol{m}_{c, \alpha}$, and in particular to draw attention to a possible difficulty in using it to study these solutions.

In the sequel we consider the one-dimensional case $N=1$ and $\alpha \in[0,1]$. Then the moving frames technique can be recast as a Hasimoto transformation as follows. Assume that $\boldsymbol{m}$ is the tangent vector of a curve in $\mathbb{R}^{3}$, i.e. $\boldsymbol{m}=\partial_{x} \boldsymbol{X}$, for some curve $\boldsymbol{X}(x, t) \in \mathbb{R}^{3}$ parametrized by the arc-length. It can be shown (see [12]) that if $\boldsymbol{m}$ evolves under $\left(\mathrm{LLG}_{\alpha}\right)$, then the torsion $\tau$ and the curvature c of $\boldsymbol{X}$ satisfy

$$
\begin{aligned}
& \partial_{t} \tau=\beta\left(\mathrm{c} \partial_{x} \mathrm{c}+\partial_{x}\left(\frac{\partial_{x x} \mathrm{c}-\mathrm{c} \tau^{2}}{\mathrm{c}}\right)\right)+\alpha\left(\mathrm{c}^{2} \tau+\partial_{x}\left(\frac{\partial_{x}(\mathrm{c} \tau)+\tau \partial_{x} \mathrm{c}}{\mathrm{c}}\right)\right), \\
& \partial_{t} \mathrm{c}=\beta\left(-\partial_{x}(\mathrm{c} \tau)-\tau \partial_{x} \mathrm{c}\right)+\alpha\left(\partial_{x} \mathrm{c}-\mathrm{c} \tau^{2}\right) .
\end{aligned}
$$

Hence, defining the Hasimoto transformation [19] (also called filament function)

$$
v(x, t)=\mathrm{c}(x, t) e^{i \int_{0}^{x} \tau(\sigma, t) d \sigma},
$$

we verify that $v$ solves the following dissipative Schrödinger (or complex Ginzburg-Landau) equation

$$
i \partial_{t} v+(\beta-i \alpha) \partial_{x x} v+\frac{v}{2}\left(\beta|v|^{2}+2 \alpha \int_{0}^{x} \operatorname{Im}\left(\bar{v} \partial_{x} v\right)-A(t)\right)=0,
$$

where $\beta=\sqrt{1-\alpha^{2}}$ and

$$
A(t)=\left(\beta\left(\mathrm{c}^{2}+\frac{2\left(\partial_{x x} \mathrm{c}-\mathrm{c} \tau^{2}\right)}{\mathrm{c}}\right)+2 \alpha\left(\frac{\partial_{x}(\mathrm{c} \tau)+\tau \partial_{x} \mathrm{c}}{\mathrm{c}}\right)\right)(0, t)
$$

The curvature and torsion associated with the self-similar solutions $\boldsymbol{m}_{c, \alpha}$ are (see [17]):

$$
\mathrm{c}_{c, \alpha}(x, t)=\frac{c}{\sqrt{t}} e^{-\frac{\alpha x^{2}}{4 t}} \quad \text { and } \quad \tau_{c, \alpha}(x, t)=\frac{\beta x}{2 \sqrt{t}} .
$$

Therefore in this case

$$
A(t)=\frac{\beta c^{2}}{t}
$$

${ }^{6}$ We recall that $v \in M^{2,2}\left(\mathbb{R}^{N}\right)$ if $v \in L_{\mathrm{loc}}^{2}\left(\mathbb{R}^{N}\right)$ and

$$
\|v\|_{M^{2,2}}:=\sup _{\substack{x \in \mathbb{R}^{N} \\ r>0}} \frac{1}{r^{(N-2) / 2}}\|v\|_{L^{2}\left(B_{r}(x)\right)}<\infty .
$$


and the Hasimoto transformation of $\boldsymbol{m}_{c, \alpha}$ is

$$
v_{c, \alpha}(x, t)=\frac{c}{\sqrt{t}} e^{(-\alpha+i \beta) \frac{x^{2}}{4 t}}
$$

In particular $v_{c, \alpha}$ is a solution of (3.36) with $A(t)$ as in (3.38), for all $\alpha \in[0,1]$ and $c>0$. Moreover, the Fourier transform of this function (w.r.t. the space variable) is

$$
\widehat{v}_{c, \alpha}(\xi, t)=2 c \sqrt{\pi(\alpha+i \beta)} e^{-(\alpha+i \beta) \xi^{2} t}
$$

so that $v_{c, \alpha}$ is a solution of (3.36) with a Dirac delta as initial condition:

$$
v_{c, \alpha}(\cdot, 0)=2 c \sqrt{\pi(\alpha+i \beta)} \delta .
$$

Here $\delta$ denotes the delta distribution at the point $x=0$ and $\sqrt{z}$ denotes the square root of a complex number $z$ such that $\operatorname{Im}(\sqrt{z})>0$.

In the limit cases $\alpha=0$ and $\alpha=1$, the first three terms in equation (3.36) lead to a cubic Schrödinger equation and to a linear heat equation, respectively. The Cauchy problem with a Dirac delta for these kind of equations associated with a power type non-linearity has been studied by several authors (see e.g. [4] and the reference therein). We recall two classical results.

Theorem $3.7([9])$. Let $p \geq 2$ and $u \in L_{\text {loc }}^{p}\left(\mathbb{R} \times \mathbb{R}^{+}\right)$be a solution in the sense of distributions of

$$
\partial_{t} u-\partial_{x x} u+|u|^{p} u=0 \quad \text { on } \mathbb{R} \times \mathbb{R}^{+} .
$$

Assume that

$$
\lim _{t \rightarrow 0^{+}} \int_{\mathbb{R}} u(x, t) \varphi(x) d x=0, \quad \text { for all } \varphi \in C_{0}(\mathbb{R} \backslash\{0\}),
$$

where $C_{0}(\mathbb{R} \backslash\{0\})$ denotes the space of continuous functions with compact support in $\mathbb{R} \backslash\{0\}$. Then $u \in C^{2,1}(\mathbb{R} \times[0, \infty))$ and $u(x, 0)=0$ for all $x \in \mathbb{R}$. In particular there is no solution of (3.39) such that

$$
\lim _{t \rightarrow 0^{+}} \int_{\mathbb{R}} u(x, t) \varphi(x) d x=\varphi(0), \quad \text { for all } \varphi \in C_{0}\left(\mathbb{R}^{N}\right) .
$$

In [9] it is also proved that if $1<p<2$, equation (3.39) has a global solution with a Dirac delta as initial condition, as in the case of the linear parabolic equation. Concerning the Schrödinger equation, we have the following ill-posedness result due to Kenig, Ponce and Vega [21].

Theorem 3.8 ([21]). Let $p \geq 2$. Either there is no solution in the sense of distributions of

$$
i \partial_{t} u+\partial_{x x} u+|u|^{p} u=0 \quad \text { on } \mathbb{R} \times \mathbb{R}^{+},
$$

with

$$
\lim _{t \rightarrow 0^{+}} u(\cdot, t)=\delta \quad \text { in } S^{\prime}(\mathbb{R}),
$$

in the class $u,|u|^{p} u \in L^{\infty}\left(\mathbb{R}^{+} ; S^{\prime}(\mathbb{R})\right)$, or there is more than one.

After performing an appropriate change of variables, equation (3.36) leads to the following equation

$$
i \partial_{t} u+(\beta-i \alpha) \partial_{x x} u+\frac{u}{2}\left(\beta|u|^{2}+2 \alpha \int_{0}^{x} \operatorname{Im}\left(\bar{u} \partial_{y} u\right) d y\right)=0 .
$$

Since $\alpha \in[0,1]$, the above equation can be seen as an intermediate model between (3.39) and (3.41). Therefore one could expect that when $\alpha \in(0,1]$, the solutions of (3.42) share similar properties to those established in Theorem 3.7 for the equation (3.39). We will continue to observe that this is not necessarily the case. To this end, we need the following: 
Proposition 3.9. For all $\alpha \in[0,1]$ and for all $\mathfrak{c} \in \mathbb{C} \backslash\{0\}$ the function $w_{\mathfrak{c}, \alpha}: \mathbb{R} \times \mathbb{R}^{+} \rightarrow \mathbb{C}$ given by

$$
w_{\mathfrak{c}, \alpha}(x, t)=\frac{\mathfrak{c}}{\sqrt{t}} \exp \left(\frac{i \beta|\mathfrak{c}|^{2}}{2} \ln (t)+(i \beta-\alpha) \frac{x^{2}}{4 t}\right)
$$

is a solution of (3.42). In addition,

i) If $\alpha \in[0,1)$, then $w_{\mathfrak{c}, \alpha}(\cdot, t)$ does not converge in $S^{\prime}(\mathbb{R})$ as $t \rightarrow 0^{+}$.

ii) If $\alpha \in(0,1]$, then

$$
\lim _{t \rightarrow 0^{+}} \int_{\mathbb{R}} w_{\mathfrak{c}, \alpha}(x, t) \varphi(x) d x=0, \quad \text { for all } \varphi \in C_{0}(\mathbb{R} \backslash\{0\}) .
$$

Proof. A straightforward computation shows that $w_{\mathfrak{c}, \alpha}$ satisfies (3.42). The proof that $w_{\mathfrak{c}, \alpha}(\cdot, t)$ does not converge in $S^{\prime}(\mathbb{R})$ as $t \rightarrow 0^{+}$if $\mathfrak{c} \neq 0$ is the same as in [21]. Indeed, for $\varphi \in S(\mathbb{R})$, by Parseval's theorem,

$$
\begin{aligned}
\int_{\mathbb{R}} \bar{w}_{\mathfrak{c}, \alpha}(x, t) \varphi(x) d x & =\frac{1}{2 \pi} \int_{\mathbb{R}} \overline{\widehat{w}}_{\mathfrak{c}, \alpha}(\xi, t) \widehat{\varphi}(\xi) d \xi \\
& =\frac{\overline{\mathfrak{c}} e^{-i \beta|\mathfrak{c}|^{2} \ln (t) / 2}}{\sqrt{\pi}} \sqrt{\alpha+i \beta} \int_{\mathbb{R}} e^{-(\alpha+i \beta) \xi^{2} t} \widehat{\varphi}(\xi) d \xi .
\end{aligned}
$$

By the dominated convergence theorem, the last integral converges:

$$
\lim _{t \rightarrow 0^{+}} \int_{\mathbb{R}} e^{-(\alpha+i \beta) \xi^{2} t} \widehat{\varphi}(\xi) d \xi=\int_{\mathbb{R}} \widehat{\varphi}(\xi) d \xi=2 \pi \varphi(0) .
$$

Since $\beta \neq 0, e^{-i \beta|\mathfrak{c}|^{2} \ln (t) / 2}$ does not admit a limit at 0 in $S^{\prime}(\mathbb{R})$. We conclude that $w_{\mathfrak{c}, \alpha}(\cdot, t)$ does not converge in $S^{\prime}(\mathbb{R})$ as $t \rightarrow 0^{+}$.

It remains to prove (3.43). Since now $\varphi \in C_{0}(\mathbb{R} \backslash\{0\})$, we cannot proceed as before. However, using the change of variables $x=\sqrt{t} y$, we have

$$
\lim _{t \rightarrow 0^{+}} \int_{\mathbb{R}} w_{\mathfrak{c}, \alpha}(x, t) \varphi(x) d x=\mathfrak{c} e^{i \beta|\mathfrak{c}|^{2} \ln (t) / 2} \int_{\mathbb{R}} e^{(-\alpha+i \beta) y^{2} / 4} \varphi(\sqrt{t} y) d y .
$$

Therefore, since $\alpha>0$ and $\varphi(0)=0$, the dominated convergence theorem implies that

$$
\lim _{t \rightarrow 0^{+}} \int_{\mathbb{R}} e^{(-\alpha+i \beta) y^{2} / 4} \varphi(\sqrt{t} y) d y=\varphi(0) \int_{\mathbb{R}} e^{(-\alpha+i \beta) y^{2} / 4} d y=0 .
$$

Since $\left|e^{i \beta|\mathbf{c}|^{2} \ln (t) / 2}\right|=1$, we obtain (3.43).

The results in Proposition 3.9 lead to the following remarks:

1. Observe that if $\alpha \in(0,1), w_{\mathfrak{c}, \alpha}$ provides a solution to the dissipative equation (3.42). Moreover, form part (ii) in Proposition 3.9, $w_{\mathfrak{c}, \alpha}$ satisfies the condition (3.40). However, notice that $w_{\mathfrak{c}, \alpha}$ cannot be extended to $C^{2,1}(\mathbb{R} \times[0, \infty))$ due to the presence of a logarithmic oscillation. This is in contrast with the properties for solutions of the cubic heat equation (3.39) established in Theorem 3.7.

2. In the case $\alpha=0$, equation (3.42) corresponds to (3.41) with $p=2$, i.e. to the equation cubic NLS equation that is invariant under the Galilean transformation. The proof of the ill-posedness result given in Theorem 3.8 relies on this invariance and part (i) of Proposition 3.9 with $\alpha=0$. Although when $\alpha>0$, equation (3.42) is no longer invariant under the Galilean transformation, part (i) of Proposition 3.9 could be an indicator that that the Cauchy problem (3.42) with a delta as initial condition is still ill-posed. This question rests open for the moment and it seems that the use of (3.36) (or (3.42)) can be more difficult to formulate a Cauchy theory for $\left(\mathrm{LLG}_{\alpha}\right)$ including self-similar solutions. 


\section{Appendix}

The characterization of $B M O_{1}^{-1}\left(\mathbb{R}^{N}\right)$ as sum of derivatives of functions in BMO was proved by Koch and Tataru in [23]. A straightforward generalization of their proof leads to the following characterization of $B M O_{\alpha}^{-1}\left(\mathbb{R}^{N}\right)$.

Theorem A.1. Let $\alpha \in(0,1]$ and $f \in S^{\prime}\left(\mathbb{R}^{N}\right)$. Then $f \in B M O_{\alpha}^{-1}\left(\mathbb{R}^{N}\right)$ if and only if there exist $f_{1}, \ldots, f_{N} \in B M O_{\alpha}\left(\mathbb{R}^{N}\right)$ such that $f=\sum_{j=1}^{N} \partial_{j} f_{j}$. In addition, if such a decomposing holds, then

$$
\|f\|_{B M O_{\alpha}^{-1}} \lesssim \sum_{j=1}^{N}\left[f_{j}\right]_{B M O_{\alpha}} .
$$

The next results provide the equivalence between the weak solutions and the Duhamel formulation. We first need to introduce for $T>0$ the space $L_{\text {uloc }}^{1}\left(\mathbb{R}^{N} \times(0, T)\right)$ defined as the space of measurable functions on $\mathbb{R}^{N} \times(0, T)$ such that the norm

$$
\|f\|_{\mathrm{uloc}, T}:=\sup _{x_{0} \in \mathbb{R}^{N}} \int_{B\left(x_{0}, 1\right)} \int_{0}^{T}|f(y, t)| d t d y
$$

is finite. We refer the reader to Lemarié-Rieusset's book [27] for more details about these kinds of spaces. In particular, we recall the following result corresponding to Lemma 11.3 in [27] in the case $\alpha=1$. It is straightforward to check that the same proof still applies if $\alpha \in(0,1)$.

Lemma A.2. Let $\alpha \in(0,1], T \in(0, \infty)$ and $w \in L_{\text {uloc }}^{1}\left(\mathbb{R}^{N} \times(0, T)\right)$. Then the function

$$
W(x, t):=\int_{0}^{t} S_{\alpha}(t-s) w(x, s) d s
$$

is well defined and belongs to $L_{\mathrm{uloc}}^{1}\left(\mathbb{R}^{N} \times(0, T)\right)$. Moreover,

$$
i \partial_{t} W+(\beta-i \alpha) \Delta W=w \quad \text { in } \quad \mathcal{D}^{\prime}\left(\mathbb{R}^{N} \times \mathbb{R}^{+}\right)
$$

and the application

$$
\begin{aligned}
{[0, T] } & \rightarrow \mathbb{R} \\
t & \mapsto\|W(\cdot, t)\|_{L^{1}\left(B_{1}\left(x_{0}\right)\right)}
\end{aligned}
$$

is continuous for any $x_{0} \in \mathbb{R}$, with $\|W(\cdot, t)\|_{L^{1}\left(B_{1}\left(x_{0}\right)\right)} \rightarrow 0$, as $t \rightarrow 0^{+}$, uniformly in $x_{0}$.

Following the ideas in [27], we can establish now the equivalence between the notions of solutions as well as the regularity.

Theorem A.3. Let $\alpha \in(0,1]$ and $u \in X\left(\mathbb{R}^{N} \times \mathbb{R}^{+} ; \mathbb{C}\right)$. Then the following assertions are equivalent:

i) The function u satisfies

$$
i u_{t}+(\beta-i \alpha) \Delta u=2(\beta-i \alpha) \frac{\bar{u}(\nabla u)^{2}}{1+|u|^{2}} \quad \text { in } \quad \mathcal{D}^{\prime}\left(\mathbb{R}^{N} \times \mathbb{R}^{+}\right) .
$$

ii) There exists $u^{0} \in \mathcal{S}^{\prime}\left(\mathbb{R}^{N}\right)$ such that $u$ satisfies

$$
u(t)=S_{\alpha}(t) u^{0}-2(\beta-i \alpha) \int_{0}^{t} S_{\alpha}(t-s) \frac{\bar{u}(\nabla u)^{2}}{1+|u|^{2}} d s .
$$


Moreover, if (ii) holds, then $u \in C^{\infty}\left(\mathbb{R}^{N} \times \mathbb{R}^{+}\right)$and

$$
\left\|\left(u(t)-u^{0}\right) \varphi\right\|_{L^{1}\left(\mathbb{R}^{N}\right)} \rightarrow 0, \quad \text { as } t \rightarrow 0^{+},
$$

for any $\varphi \in \mathcal{S}\left(\mathbb{R}^{N}\right)$.

Proof. In view of Lemma A.2, we need to prove that the function

$$
g(u)=-2(\beta-i \alpha) \frac{\bar{u}(\nabla u)^{2}}{1+|u|^{2}}
$$

belongs to $L_{\text {uloc }}^{1}\left(\mathbb{R}^{N} \times(0, T)\right)$, for all $T>0$. Indeed, by (2.3) we have

$$
\|g(u)\|_{\mathrm{uloc}, T} \leq\left\||\nabla u|^{2}\right\|_{\mathrm{uloc}, T}
$$

If $T \leq 1$, then

$$
\left\||\nabla u|^{2}\right\|_{\mathrm{uloc}, T} \leq \sup _{x_{0} \in \mathbb{R}^{N}} \int_{Q_{1}\left(x_{0}\right)}|\nabla u(y, t)|^{2} d t d y \leq\|u\|_{X}^{2} .
$$

If $T \geq 1$, using that

$$
|\nabla u| \leq \frac{[u]_{X}}{\sqrt{t}}, \quad \text { for any } t>0
$$

we get

$$
\begin{aligned}
\left\||\nabla u|^{2}\right\|_{T, \text { uloc }} & \leq \sup _{x_{0} \in \mathbb{R}^{N}} \int_{Q_{1}\left(x_{0}\right)}|\nabla u(y, t)|^{2} d t d y+\sup _{x_{0} \in \mathbb{R}^{N}} \int_{1}^{T} \int_{B_{1}\left(x_{0}\right)}|\nabla u|^{2} d y d t \\
& \leq\|u\|_{X}^{2}+[u]_{X}^{2}\left|B_{1}(0)\right| \int_{1}^{T} \frac{1}{t} d t \\
& \leq\|u\|_{X}^{2}\left(1+\left|B_{1}(0)\right| \ln (T)\right) .
\end{aligned}
$$

In conclusion, we deduce from (A.3), (A.4) and (A.5) that $g(u) \in L_{\text {uloc }}^{1}\left(\mathbb{R}^{N} \times(0, T)\right)$ and then it follows from Lemma A.2 that (ii) implies (i). The other implication can be established as in [27, Theorem 11.2]. Moreover, we deduce that the function

$$
W(x, t):=T(g(u))(x, t)=\int_{0}^{t} S_{\alpha}(t-s) g(u) d s
$$

satisfies $\|W(\cdot, t)\|_{L^{1}\left(B_{1}\left(x_{0}\right)\right)} \rightarrow 0$, as $t \rightarrow 0^{+}$, uniformly in $x_{0} \in \mathbb{R}^{N}$. Let us take $\varphi \in S\left(\mathbb{R}^{N}\right)$ and a constant $C_{\varphi}>0$ such that $|\varphi(x)| \leq C_{\varphi}(2+|x|)^{-N-1}$. Then

$$
\begin{aligned}
\int_{\mathbb{R}^{N}}|\varphi(y) W(y, t)| d y & \leq \sum_{k \in \mathbb{Z}^{N}} \int_{B_{1}(k)} \frac{C_{\varphi}}{(2+|x|)^{N+1}}|W(y, t)| d y \\
& \leq \sup _{x_{0} \in \mathbb{R}^{N}}\|W(\cdot, t)\|_{L^{1}\left(B_{1}\left(x_{0}\right)\right)} \sum_{k \in \mathbb{Z}^{N}} \frac{C_{\varphi}}{(1+|k|)^{N+1}},
\end{aligned}
$$

so that $\|\varphi W(\cdot, t)\|_{L^{1}\left(\mathbb{R}^{N}\right)} \rightarrow 0$ as $t \rightarrow 0^{+}$, i.e.

$$
\left\|\left(u(t)-S_{\alpha}(t) u^{0}\right) \varphi\right\|_{L^{1}\left(\mathbb{R}^{N}\right)} \rightarrow 0, \quad \text { as } t \rightarrow 0^{+} .
$$

On the other hand, since $u^{0} \in L^{\infty}\left(\mathbb{R}^{N}\right)$,

$$
\left\|S_{\alpha}(t) u^{0}-u^{0}\right\|_{L^{1}\left(B_{r}(0)\right)} \rightarrow 0, \quad \text { as } t \rightarrow 0^{+},
$$


for any $r>0$ (see e.g. [3, Corollary 2.4]). Given $\epsilon>0$, we fix $r_{\epsilon}>0$ such that

$$
2\left\|u^{0}\right\|_{\infty}\|\varphi\|_{L^{1}\left(B_{r_{\epsilon}}^{c}(0)\right)} \leq \epsilon .
$$

Using (A.7), we obtain

$$
\lim _{t \rightarrow 0^{+}}\left\|\left(S_{\alpha}(t) u^{0}-u^{0}\right) \varphi\right\|_{L^{1}\left(B_{r_{\epsilon}}(0)\right)}=0 .
$$

Then, passing to limit in the inequality

$$
\left\|\left(S_{\alpha}(t) u^{0}-u^{0}\right) \varphi\right\|_{L^{1}\left(\mathbb{R}^{N}\right)} \leq\left\|\left(S_{\alpha}(t) u^{0}-u^{0}\right) \varphi\right\|_{L^{1}\left(B_{r_{\epsilon}}(0)\right)}+2\left\|u^{0}\right\|_{L^{\infty}\left(\mathbb{R}^{N}\right)}\|\varphi\|_{L^{1}\left(B_{r_{\epsilon}}^{c}(0)\right)},
$$

we obtain

$$
\limsup _{t \rightarrow 0^{+}}\left\|\left(S_{\alpha}(t) u^{0}-u^{0}\right) \varphi\right\|_{L^{1}\left(\mathbb{R}^{N}\right)} \leq \epsilon
$$

Therefore

$$
\lim _{t \rightarrow 0^{+}}\left\|\left(S_{\alpha}(t) u^{0}-u^{0}\right) \varphi\right\|_{L^{1}\left(\mathbb{R}^{N}\right)}=0 .
$$

Combining with (A.6), we conclude the proof of (A.2).

It remains to prove that $u$ is smooth for $t>0$. Since $u \in X\left(\mathbb{R}^{N} \times \mathbb{R}^{+} ; \mathbb{C}\right)$, we get that $u, \nabla u \in L_{\text {loc }}^{\infty}\left(\mathbb{R}^{N} \times \mathbb{R}^{+}\right)$. Then $g(u) \in L_{\text {loc }}^{2}\left(\mathbb{R}^{N} \times \mathbb{R}^{+}\right)$so the $L^{p}$-regularity theory for parabolic equations implies that a function $u$ satisfying (A.1) belongs to $u \in H_{\text {loc }}^{2,1}\left(\mathbb{R}^{N} \times \mathbb{R}^{+}\right.$) (see $[28,24]$ and [33, Remark 48.3] for notations and more details). Since the space $H^{k} \cap L^{\infty}$ is stable under multiplication (see e.g. [20, Chapter 6]), we can use a bootstrap argument to conclude that $u \in C^{\infty}\left(\mathbb{R}^{N} \times \mathbb{R}^{+}\right)$.

Remark A.4. Several authors have studied further properties of the solutions found by Koch and Tataru for the Navier-Stokes equations. For instance, analyticity, decay rates of the higherorder derivatives in space and time have been investigated by Miura and Sawada [32], Germain, Pavlović and Staffilani [14], among others. A similar analysis for the solution $u$ of (DNLS) is beyond the scope of this paper, but it can probably be performed using the same arguments given in $[32,14]$.

We end this appendix with some properties of the self-similar found in [17].

Theorem A.5 ([17]). Let $N=1$. For every $\alpha \in[0,1]$ and $c>0$, there exists a profile $\boldsymbol{f}_{c, \alpha} \in$ $C^{\infty}\left(\mathbb{R}, \mathbb{S}^{2}\right)$ such that

$$
\boldsymbol{m}_{c, \alpha}(x, t)=\boldsymbol{f}_{c, \alpha}\left(\frac{x}{\sqrt{t}}\right), \quad \text { for all }(x, t) \in \mathbb{R} \times \mathbb{R}^{+},
$$

is a smooth solution of $\left(\mathrm{LLG}_{\alpha}\right)$ on $\mathbb{R} \times \mathbb{R}^{+}$. Moreover,

(i) There exist unitary vectors $\boldsymbol{A}_{c, \alpha}^{ \pm}=\left(A_{j, c, \alpha}^{ \pm}\right)_{j=1}^{3} \in \mathbb{S}^{2}$ such that the following pointwise convergence holds when $t$ goes to zero:

$$
\lim _{t \rightarrow 0^{+}} \boldsymbol{m}_{c, \alpha}(x, t)= \begin{cases}\boldsymbol{A}_{c, \alpha}^{+}, & \text {if } x>0, \\ \boldsymbol{A}_{c, \alpha}^{-}, & \text {if } x<0,\end{cases}
$$

and $\boldsymbol{A}_{c, \alpha}^{-}=\left(A_{1, c, \alpha}^{+},-A_{2, c, \alpha}^{+},-A_{3, c, \alpha}^{+}\right)$.

(ii) There exists a constant $C(c, \alpha, p)$, depending only on $c, \alpha$ and $p$ such that for all $t>0$

$$
\left\|\boldsymbol{m}_{c, \alpha}(\cdot, t)-\boldsymbol{A}_{c, \alpha}^{+} \chi_{(0, \infty)}(\cdot)-\boldsymbol{A}_{c, \alpha}^{-} \chi_{(-\infty, 0)}(\cdot)\right\|_{L^{p}(\mathbb{R})} \leq C(c, \alpha, p) t^{\frac{1}{2 p}},
$$

for all $p \in(1, \infty)$. In addition, if $\alpha>0$, (A.11) also holds for $p=1$. 
(iii) For $t>0$ and $x \in \mathbb{R}$, the derivative in space satisfies

$$
\left|\partial_{x} \boldsymbol{m}_{c, \alpha}(x, t)\right|=\frac{c}{\sqrt{t}} e^{-\frac{\alpha x^{2}}{4 t}} .
$$

(iv) Let $\alpha \in[0,1]$. Then $\boldsymbol{A}_{c, \alpha}^{+} \rightarrow(1,0,0)$ as $c \rightarrow 0^{+}$.

Lemma A.6. Let $c>0, \alpha \in(0,1], \boldsymbol{A}_{c, \alpha}^{+}, \boldsymbol{A}_{c, \alpha}^{-}$be the unit vectors given in Theorem A.5 and $\vartheta_{c, \alpha}$ the angle between $\boldsymbol{A}_{c, \alpha}^{+}$and $\boldsymbol{A}_{c, \alpha}^{-}$. Then, for fixed $\alpha \in(0,1], \vartheta_{c, \alpha}$ is a continuous function in c. Also, for $0<c<\alpha^{2} \sqrt{\pi} / 32$,

$$
\vartheta_{c, \alpha} \geq \arccos \left(1-c^{2} \pi+\frac{32 c^{3} \sqrt{\pi}}{\alpha^{2}}\right) .
$$

Remark A.7. If $\alpha \in(0,1]$ and $c \in\left(0, \alpha^{2} \sqrt{\pi} / 32\right)$, then $1-c^{2} \pi+\frac{32 c^{3} \sqrt{\pi}}{\alpha^{2}} \in(0,1)$, so that its arccos is well-defined.

Proof. The continuity was proved in [17]. To show the estimate (A.13), we use Theorem 1.3 in [17], to get

$$
\left|A_{2, c, \alpha}^{+}-c \frac{\sqrt{\pi(1+\alpha)}}{\sqrt{2}}\right| \leq \frac{c^{2} \pi}{4}+\frac{c^{2} \pi}{\alpha \sqrt{2}}\left(1+\frac{c^{2} \pi}{8}+c \frac{\sqrt{\pi(1+\alpha)}}{2 \sqrt{2}}\right)+\left(\frac{c^{2} \pi}{2 \sqrt{2} \alpha}\right)^{2} .
$$

Since $\alpha \in(0,1]$, we have for any $c \in(0,1]$,

$$
\frac{\pi}{4}+\frac{\pi}{\alpha \sqrt{2}}\left(1+\frac{c^{2} \pi}{8}+c \frac{\sqrt{\pi(1+\alpha)}}{2 \sqrt{2}}\right)+\frac{c^{2} \pi^{2}}{8 \alpha^{2}} \leq \frac{1}{\alpha^{2}}\left(\frac{\pi}{4}+\frac{\pi}{\sqrt{2}}\left(1+\frac{\pi}{8}+\frac{\sqrt{\pi}}{2}\right)+\frac{\pi^{2}}{8}\right) \leq \frac{8}{\alpha^{2}} .
$$

We deduce that for all $\alpha, c \in(0,1]$,

$$
A_{2, c, \alpha}^{+} \geq c \frac{\sqrt{\pi(1+\alpha)}}{\sqrt{2}}-\frac{8 c^{2}}{\alpha^{2}} \geq c \frac{\sqrt{\pi}}{\sqrt{2}}-\frac{8 c^{2}}{\alpha^{2}} .
$$

In particular $A_{2, c, \alpha}^{+} \geq 0$ if $c \leq \alpha^{2} \sqrt{\pi} /(8 \sqrt{2})$. Thus

$$
\left(A_{2, c, \alpha}^{+}\right)^{2} \geq \frac{c^{2} \pi}{2}-\frac{16 c^{3} \sqrt{\pi}}{\sqrt{2} \alpha^{2}}+\frac{64 c^{4}}{\alpha^{4}} \geq \frac{c^{2} \pi}{2}-\frac{16 c^{3} \sqrt{\pi}}{\alpha^{2}},
$$

so that

$$
\cos \left(\vartheta_{c, \alpha}\right)=1-2\left(\left(A_{2, c, \alpha}^{+}\right)^{2}+\left(A_{3, c, \alpha}^{+}\right)^{2}\right) \leq 1-c^{2} \pi+\frac{32 c^{3} \sqrt{\pi}}{\alpha^{2}},
$$

which implies (A.13).

The following lemma is a slightly refinement of Theorem 1.4 in [17].

Lemma A.8 ([17]). Let $c>0, \alpha \in[0,1]$ and $\boldsymbol{A}_{c, \alpha}^{+}$be the unit vector given in Theorem A.5. Then $\boldsymbol{A}_{c, \alpha}^{+}$is a continuous function of $\alpha$ in $[0,1]$ and

$$
\left|\boldsymbol{A}_{c, \alpha}^{+}-\boldsymbol{A}_{c, 1}^{+}\right| \leq h(c) \sqrt{1-\alpha}, \quad \text { for all } \alpha \in[1 / 2,1]
$$

where $h: \mathbb{R}^{+} \rightarrow \mathbb{R}^{+}$is a strictly increasing function satisfying

$$
\lim _{s \rightarrow \infty} h(s)=\infty
$$


Proof. In view of [17, Theorem 1.4], we only need to prove that the constant $C(c)$ in the statement of the Theorem 1.4 (notice that $c_{0}$ in [17] corresponds to $c$ in our notation) is polynomial in $c$ with nonnegative coefficients. Looking at the proof of [17, Theorem 1.4], we see that the constant $C(c)$ behaves like the constant in inequality (3.108) in [17]. In view of (3.17), the estimate (3.23) in [17] can be written as

$$
|f(s)| \leq \sqrt{2} \text { and }\left|f^{\prime}(s)\right| \leq \frac{c}{2} e^{-\alpha s^{2} / 4},
$$

and then (3.18) can be recast as

$$
|g| \leq\left(\frac{c}{4}+\frac{c^{2} \sqrt{2}}{8}\right)\left(\frac{s}{\beta} e^{-\alpha s^{2} / 4}+s^{2} e^{-\alpha s^{2} / 2}\right) .
$$

Then, it can be easily checked that the function $h$ is a polynomial with nonnegative coefficients.

Acknowledgments. A. de Laire was partially supported by the Labex CEMPI (ANR-11-LABX0007-01) and the MathAmSud program. S. Gutierrez was partially supported by the EPSRC grant EP/J01155X/1 and the ERCEA Advanced Grant 2014669689 - HADE.

\section{References}

[1] M. Abramowitz and I. A. Stegun. Handbook of mathematical functions with formulas, graphs, and mathematical tables, volume 55 of National Bureau of Standards Applied Mathematics Series. For sale by the Superintendent of Documents, U.S. Government Printing Office, Washington, D.C., 1964.

[2] F. Alouges and A. Soyeur. On global weak solutions for Landau-Lifshitz equations: existence and nonuniqueness. Nonlinear Anal., 18(11):1071-1084, 1992.

[3] J. M. Arrieta, A. Rodriguez-Bernal, J. W. Cholewa, and T. Dlotko. Linear parabolic equations in locally uniform spaces. Math. Models Methods Appl. Sci., 14(2):253-293, 2004.

[4] V. Banica and L. Vega. On the Dirac delta as initial condition for nonlinear Schrödinger equations. Ann. Inst. H. Poincaré Anal. Non Linéaire, 25(4):697-711, 2008.

[5] V. Banica and L. Vega. On the stability of a singular vortex dynamics. Comm. Math. Phys., 286(2):593-627, 2009.

[6] V. Banica and L. Vega. Scattering for 1D cubic NLS and singular vortex dynamics. J. Eur. Math. Soc. (JEMS), 14(1):209-253, 2012.

[7] V. Banica and L. Vega. Stability of the self-similar dynamics of a vortex filament. Arch. Ration. Mech. Anal., 210(3):673-712, 2013.

[8] P. Biernat and P. Bizoń. Shrinkers, expanders, and the unique continuation beyond generic blowup in the heat flow for harmonic maps between spheres. Nonlinearity, 24(8):2211-2228, 2011.

[9] H. Brezis and A. Friedman. Nonlinear parabolic equations involving measures as initial conditions. J. Math. Pures Appl. (9), 62(1):73-97, 1983. 
[10] H. Brezis and L. Nirenberg. Degree theory and BMO. I. Compact manifolds without boundaries. Selecta Math. (N.S.), 1(2):197-263, 1995.

[11] J.-M. Coron. Nonuniqueness for the heat flow of harmonic maps. Ann. Inst. H. Poincaré Anal. Non Linéaire, 7(4):335-344, 1990.

[12] M. Daniel and M. Lakshmanan. Perturbation of solitons in the classical continuum isotropic Heisenberg spin system. Physica A: Statistical Mechanics and its Applications, 120(1):125$152,1983$.

[13] S. Ding and C. Wang. Finite time singularity of the Landau-Lifshitz-Gilbert equation. Int. Math. Res. Not. IMRN, (4):Art. ID rnm012, 25, 2007.

[14] P. Germain, N. Pavlović, and G. Staffilani. Regularity of solutions to the Navier-Stokes equations evolving from small data in $\mathrm{BMO}^{-1}$. Int. Math. Res. Not. IMRN, (21):Art. ID rnm087, 35, 2007.

[15] P. Germain and M. Rupflin. Selfsimilar expanders of the harmonic map flow. Ann. Inst. H. Poincaré Anal. Non Linéaire, 28(5):743-773, 2011.

[16] T. L. Gilbert. A lagrangian formulation of the gyromagnetic equation of the magnetization field. Phys. Rev., 100:1243, 1955.

[17] S. Gutiérrez and A. de Laire. Self-similar solutions of the one-dimensional Landau-LifshitzGilbert equation. Nonlinearity, 28(5):1307, 2015.

[18] S. Gutiérrez, J. Rivas, and L. Vega. Formation of singularities and self-similar vortex motion under the localized induction approximation. Comm. Partial Differential Equations, 28(56):927-968, 2003.

[19] H. Hasimoto. A soliton on a vortex filament. J. Fluid Mech, 51(3):477-485, 1972.

[20] L. Hörmander. Lectures on nonlinear hyperbolic differential equations, volume 26 of Mathématiques 85 Applications (Berlin) [Mathematics $\&$ Applications]. Springer-Verlag, Berlin, 1997.

[21] C. E. Kenig, G. Ponce, and L. Vega. On the ill-posedness of some canonical dispersive equations. Duke Math. J., 106(3):617-633, 2001.

[22] H. Koch and T. Lamm. Geometric flows with rough initial data. Asian J. Math., 16(2):209$235,2012$.

[23] H. Koch and D. Tataru. Well-posedness for the Navier-Stokes equations. Adv. Math., 157(1):22-35, 2001.

[24] O. Ladyzhenskaya, V. Solonnikov, and N. Ural'tseva. Linear and quasi-linear equations of parabolic type. Amer. Math. Soc., Transl. Math. Monographs. Providence, R.I., 1968.

[25] M. Lakshmanan and K. Nakamura. Landau-Lifshitz equation of ferromagnetism: Exact treatment of the Gilbert damping. Phys. Rev. Lett., 53:2497-2499, 1984.

[26] L. Landau and E. Lifshitz. On the theory of the dispersion of magnetic permeability in ferromagnetic bodies. Phys. Z. Sowjetunion, 8:153-169, 1935.

[27] P. G. Lemarié-Rieusset. Recent developments in the Navier-Stokes problem, volume 431 of Chapman $8 \mathrm{Hall} / \mathrm{CRC}$ Research Notes in Mathematics. Chapman \& Hall/CRC, Boca Raton, FL, 2002. 
[28] G. M. Lieberman. Second order parabolic differential equations. World Scientific Publishing Co., Inc., River Edge, NJ, 1996.

[29] J. Lin. Uniqueness of harmonic map heat flows and liquid crystal flows. Discrete Contin. Dyn. Syst., 33(2):739-755, 2013.

[30] J. Lin, B. Lai, and C. Wang. Global well-posedness of the Landau-Lifshitz-Gilbert equation for initial data in Morrey spaces. Calc. Var. Partial Differential Equations, 54(1):665-692, 2015.

[31] C. Melcher. Global solvability of the Cauchy problem for the Landau-Lifshitz-Gilbert equation in higher dimensions. Indiana Univ. Math. J., 61(3):1175-1200, 2012.

[32] H. Miura and O. Sawada. On the regularizing rate estimates of Koch-Tataru's solution to the Navier-Stokes equations. Asymptot. Anal., 49(1-2):1-15, 2006.

[33] P. Quittner and P. Souplet. Superlinear parabolic problems. Birkhäuser Advanced Texts: Basler Lehrbücher. [Birkhäuser Advanced Texts: Basel Textbooks]. Birkhäuser Verlag, Basel, 2007. Blow-up, global existence and steady states.

[34] E. M. Stein. Harmonic analysis: real-variable methods, orthogonality, and oscillatory integrals, volume 43 of Princeton Mathematical Series. Princeton University Press, Princeton, NJ, 1993. With the assistance of Timothy S. Murphy, Monographs in Harmonic Analysis, III.

[35] C. Wang. Well-posedness for the heat flow of harmonic maps and the liquid crystal flow with rough initial data. Arch. Ration. Mech. Anal., 200(1):1-19, 2011.

[36] D. Wei. Micromagnetics and Recording Materials. SpringerBriefs in Applied Sciences and Technology. Springer Berlin Heidelberg, 2012. 